\title{
CONSTRAINED BEST EUCLIDEAN DISTANCE EMBEDDING ON A SPHERE: A MATRIX OPTIMIZATION APPROACH*
}

\author{
SHUANGHUA BAI ${ }^{\dagger}$, HOU-DUO $\mathrm{QI}^{\ddagger}$, AND NAIHUA XIU§
}

\begin{abstract}
The problem of data representation on a sphere of unknown radius arises from various disciplines such as Statistics (spatial data representation), Psychology (constrained multidimensional scaling), and Computer Science (machine learning and pattern recognition). The best representation often needs to minimize a distance function of the data on a sphere as well as to satisfy some Euclidean distance constraints. It is those spherical and Euclidean distance constraints that present an enormous challenge to the existing algorithms. In this paper, we reformulate the problem as an Euclidean distance matrix optimization problem with a low rank constraint. We then propose an iterative algorithm that uses a quadratically convergent Newton-CG method at its each step. We study fundamental issues including constraint nondegeneracy and the nonsingularity of generalized Jacobian that ensure the quadratic convergence of the Newton method. We use some classic examples from the spherical multidimensional scaling to demonstrate the flexibility of the algorithm in incorporating various constraints. We also present an interesting application to the circle fitting problem.
\end{abstract}

Key words. Euclidean distance matrix, Matrix optimization, Lagrangian duality, Spherical multidimensional scaling, Semismooth Newton-CG method.

AMS subject classifications. 49M45, 90C25, 90C33

1. Introduction. In this paper, we are mainly concerned with placing $n$ points $\left\{x^{1}, \ldots, x^{n}\right\}$ in a best way on a sphere in $\mathbb{R}^{r}$. The primary information that we use is an incomplete/complete set of pairwise Euclidean distances (often with noises) among the $n$ points. In such a setting, $\mathbb{R}^{r}$ is often a low-dimensional space (e.g., $r$ takes 2 or 3 for data visualization) and is known as the embedding space. The center of the sphere is unknown. For some applications, the center can be put at origin in $\mathbb{R}^{r}$. Furthermore, the radius of the sphere is also unknown. In our matrix optimization formulation of the problem, we treat both the center and the radius as unknown variables. We develop a fast numerical method for this problem and present a few of interesting applications taken from existing literature.

The problem described above has long appeared in the constrained Multi-Dimensional Scaling (MDS) when $r \leq 3$, which is mainly for the purpose of data visualization, see [9, Sect. 4.6] and [4, Sect. 10.3] for more details. In particular, it is known as the spherical MDS when $r=3$ and the circular MDS when $r=2$. Most numerical methods in this part took advantages of $r$ being 2 or 3. For example, two of the earliest circular MDS were by Borg and Lingoes [5] and Lee and Bentler [28], where they introduced a new point $x^{0} \in \mathbb{R}^{r}$ as the center of the sphere (i.e., circles in their case) and further forced the following constraints to hold:

$$
d_{01}=d_{02}=\cdots=d_{0 n} .
$$

Here $d_{0 j}=\left\|x^{0}-x^{j}\right\|, j=1, \ldots, n$ are the Euclidean distances between the center $x^{0}$ and the other $n$ points. In their models, the variables are the coordinates of the

*December 23, 2013; Revised July 20, 2014; Accepted December 1, 2014.

†School of Mathematics, The University of Southampton, Highfield, Southampton SO17 1BJ, UK. (sb11g12@soton.ac.uk.),

${ }^{\ddagger}$ School of Mathematics, The University of Southampton, Highfield, Southampton SO17 1BJ, UK. (hdqi@soton.ac.uk.) The author was supported in part by the EPSRC grant EP/K007645/1.

$\S$ Department of Applied Mathematics, Beijing Jiaotong University, Beijing, China. (naihua_xiu@126.com.) The author was supported in part by NSFC grants 71271021 and 11431002. 
$(n+1)$ points in $\mathbb{R}^{r}$. In [5], the optimal criterion was a stress function widely used in MDS literature (see [4, Chp. 3]), whereas [28] used a least square loss function as its optimal criterion.

In the spherical MDS of [10], Cox and Cox placed the center of the sphere at origin and represented the $n$ points by their spherical coordinates. Moreover, they also argued for the Euclidean distance to be used over the seemingly more appropriate geodesic distance on the sphere. This is particularly the case when the order of the distances among the $n$ points are more important than the magnitude of their actual distances. For the accurate relationship between Euclidean distance and the geodesic distance on a sphere, see [38, Thm. 3.23], which is credited to Schoenberg [47]. A recent method known as MDS on a quadratic surface (MDS-Q) was proposed by de Leeuw and Mair [13], where geodesic distances were used. As noted in [13, p. 12], "geodesic MDS-Q, however, seems limited for now to spheres in any dimension, with the possible exception of ellipses and parabolas in $\mathbb{R}^{2}$ ". For the spherical case, MDS$\mathrm{Q}$ places the center at origin and the variables are the radius and the coordinates of the $n$ points on the sphere. The Euclidean distances were then converted to the corresponding geodesic distances. The optimal criterion is a weighted least square loss function.

When the center of the sphere is placed at origin, any point on the sphere satisfies the spherical constraint of the type $\|x\|=R$, where $x \in \mathbb{R}^{r}$ and $R$ is the radius. Optimization with spherical constraints has recently attracted much attention of researchers, see, e.g., [32, 31, 16, 17, 29, 55] and the references therein. Such a problem can be cast as a more general optimization problem over the Stiefel manifold $[52,25]$. One important example is the nearest low-rank correlation matrix problem, where the unit diagonals of the correlation matrix yields the spherical constraints $[17,29,52,25]$. It is noted that the sequential second-order methods in $[17,29]$ as well as the feasibility-preserving methods in $[52,25]$ all rely on the fact that the radius is known (e.g., $R=1$ ). This is in contrast to our problem where $R$ is a variable.

In this paper, we propose a matrix optimization formulation that is conducive to theoretical investigation and design of (second-order) numerical methods. An important concept that we will use is the Euclidean Distance Matrix (EDM), which will be the matrix variable in our formulation. Hence, our optimization problem can be treated as an Euclidean distance problem, recently surveyed by Liberti et al. [30]. EDM optimization has been extensively studied and found many applications including sensor network localization and molecular confirmation, see, e.g., $[20,11,15,18,19,36,12,51,35,27,39,41]$. However, it appears that none of the existing EDM optimization methods known to us can be directly applied to handle the spherical constraints with an unknown radius. We also like to point out that, though similar in structure, the obtained EDM optimization is more involved (having more spherical constraints) than those problems studied in the previous research $[39,41]$, where only the simple diagonal constraints were studied. The implication is that we have to develop a whole set of different techniques and analysis for our new EDM optimization problem. For example, the important property of constraint nondegeneracy does not automatically hold any more and its analysis is much more complicated. We regard this as an important technical contribution in this paper.

The paper is organized as follows with the main contributions highlighted. In Sect. 2, We first argue that when the EDM is used to formulate the problem, it is necessary to introduce a new point to represent the center of the sphere. This is due to a special property arising from embedding an EDM. The algorithmic framework 
that we use for the obtained non-convex matrix optimization problem is closely related to the majorized penalty method of Gao and Sun [17] for the nearest low-rank correlation matrix problem. One of the key elements in this type of method is that the subproblems are convex. Those convex problems are structurally similar to a convex relaxation of the original matrix optimization problem and they all can be solved by a quadratically convergent Newton-CG method. We establish that this is the case for our problem by studying the challenging issue of constraint nondegeneracy, which further ensures the nonsingularity of generalized Jacobian used by the Newton-CG method. Those results can be found in Sect. 3 and ensure that the extension of the majorization method of Gao and Sun [17] to our problem is complete. The algorithm is presented in Sect. 4 and its key convergent results are stated without detailed proofs as they can be proved similarly as in [17]. Sect. 5 aims to demonstrate a variety of applications from classical MDS to the circle fitting problem. The numerical performance is highly satisfactory with those applications. We conclude in Sect. 6 .

Notation. Let $\mathcal{S}^{n}$ denote the space of $n \times n$ symmetric matrices equipped with the standard inner product $\langle A, B\rangle=\operatorname{Tr}(A B)$ for $A, B \in \mathcal{S}^{n}$. Let $\|\cdot\|$ denote the induced Frobenius norm. Let $\mathcal{S}_{+}^{n}$ denote the cone of positive semidefinite matrices in $\mathcal{S}^{n}$ (often abbreviated as $X \succeq 0$ for $X \in \mathcal{S}_{+}^{n}$ ). The so-called hollow subspace $\mathcal{S}_{h}^{n}$ is defined by (":=" means define)

$$
\mathcal{S}_{h}^{n}:=\left\{A \in \mathcal{S}^{n}: \operatorname{diag}(A)=0\right\},
$$

where $\operatorname{diag}(A)$ is the vector formed by the diagonal elements of $A$. For subsets $\alpha, \beta$ of $\{1, \ldots, n\}$, denote $A_{\alpha \beta}$ as the submatrix of $A$ indexed by $\alpha$ and $\beta$ ( $\alpha$ for rows and $\beta$ for columns). $A_{\alpha}$ denotes the submatrix consisting of columns of $A$ indexed by $\alpha$, and $|\alpha|$ is the cardinality of $\alpha$. Throughout the paper, vectors are treated as column vectors. For example, $x^{T}$ is a row vector for $x \in \mathbb{R}^{n}$. The vector $e$ is the vector of all ones and $I$ denotes the identity matrix, whose dimension is clear from the context. When it is necessary, we use $I_{n}$ to indicate its dimension $n$. Let $e_{i}$ denote the $i$ th unit vector, which is the $i$ th column of $I$. Let $Q$ be the Householder transformation that maps $e \in \mathbb{R}^{n+1}$ to the vector $[0, \ldots, 0,-\sqrt{n+1}]^{T} \in \mathbb{R}^{n+1}$. Let

$$
v:=[1, \ldots, 1,1+\sqrt{n+1}]^{T}=e+\sqrt{n+1} e_{n+1} .
$$

Then

$$
Q=I_{n+1}-\frac{2}{v^{T} v} v v^{T}
$$

Let

$$
J:=I_{n+1}-\frac{1}{n+1} e e^{T} .
$$

We often use the following properties:

$$
J^{2}=J, \quad Q^{2}=I \quad \text { and } \quad J=Q\left[\begin{array}{cc}
I_{n} & 0 \\
0 & 0
\end{array}\right] Q .
$$

2. The Matrix Optimization Formulation. In this section, we first give a brief review of the important concept EDM and its relevant properties. We then apply EDM to our problem to derive the matrix optimization formulation. 
2.1. Background on EDM. There are three elements that have become basics in the research of Euclidean distance embedding. The first one is the definition of the squared Euclidean distance matrix (EDM). The second are various characterizations of EDMs. And the third one is the Procrustes analysis that produces actual embedding in an Euclidean space. We briefly describe them one by one. Standard references are $[9,4,12]$. Throughout, we use dimension $(n+1)$, which is more convenient for our application. Of course, all results in this subsection can be stated in dimension $n$.

(a) Squared EDM. A matrix $D$ is a (squared) EDM if $D \in \mathcal{S}_{h}^{n+1}$ and there exist points $x^{1}, \ldots, x^{n+1}$ in $\mathbb{R}^{r}$ such that $D_{i j}=\left\|x^{i}-x^{j}\right\|^{2}$ for $i, j=1, \ldots, n+1$. $\mathbb{R}^{r}$ is often referred to as the embedding space and $r$ is the embedding dimension when it is the smallest such $r$. We note that $D$ must belong to $\mathcal{S}_{h}^{n+1}$ if it is an EDM. Furthermore, if those $\left\{x^{i}\right\}$ lie on a sphere, $D$ is called an EDM on a sphere.

(b) Characterizations of EDM. It is well-known that a matrix $D \in \mathcal{S}^{n+1}$ is an EDM if and only if

$$
D \in \mathcal{S}_{h}^{n+1} \text { and } \quad J(-D) J \succeq 0 .
$$

The origin of this result can be traced back to Schoenberg [46] and an independent work [53] by Young and Householder. See also Gower [21] for a nice derivation of (2). Moreover, the corresponding embedding dimension is $r=\operatorname{rank}(J D J)$.

It is noted that the matrix $J$, when treated as an operator, is the orthogonal projection onto the subspace $e^{\perp}:=\left\{x \in \mathbb{R}^{n+1}: e^{T} x=0\right\}$. Characterization (2) simply means that $D$ is an EDM if and only if $D \in \mathcal{S}_{h}^{n+1}$ and $D$ is negative semidefinite on the subspace $e^{\perp}$ :

$$
-D \in \mathcal{K}_{+}^{n+1}:=\left\{A \in \mathcal{S}^{n+1}: x^{T} A x \geq 0, \forall x \in \mathbb{R}^{n+1} \text { such that }\langle x, e\rangle=0\right\} .
$$

It follows that $\mathcal{K}_{+}^{n+1}$ is a closed convex cone (known as the almost positive semidefinite cone). Let $\Pi_{\mathcal{K}_{+}^{n+1}}(X)$ denote the orthogonal projection of $X \in \mathcal{S}^{n+1}$ onto $\mathcal{K}_{+}^{n+1}$ :

$$
\Pi_{\mathcal{K}_{+}^{n+1}}(X):=\arg \min \|X-Y\| \quad \text { s.t } \quad Y \in \mathcal{K}_{+}^{n+1} .
$$

A nice property is that this projection can be done through the orthogonal projection onto the positive semidefinite cone $\mathcal{S}_{+}^{n+1}$ and is due to Gaffke and Mathar [15]

$$
\Pi_{\mathcal{K}_{+}^{n+1}}(X)=X+\Pi_{\mathcal{S}_{+}^{n+1}}(-J X J) \quad \forall X \in \mathcal{S}^{n+1} .
$$

(c) Euclidean Embedding. If $D$ is an EDM with embedding dimension $r$, then $-J D J \succeq 0$ by (2). Let

$$
-J D J / 2=X^{T} X
$$

where $X \in \mathbb{R}^{r \times(n+1)}$. Let $x^{i}$ denote the $i$ th column of $X$. It is known that $\left\{x^{1}, \ldots, x^{n+1}\right\}$ are the embedding points of $D$ in $\mathbb{R}^{r}$, i.e., $D_{i j}=\left\|x^{i}-x^{j}\right\|^{2}$. We also note that any rotation and shifting of $\left\{x^{1}, \ldots, x^{n+1}\right\}$ would give same $D$. In other words, there are infinitely many sets of embedding points. To find a desired set of embedding points that match positions of certain existing points, one needs to conduct the Procrustes analysis, which is a computational scheme and often has a 
closed-form formula, see [9, Chp. 5]. We omit the details here. It is also important to point out that in many applications, any set of embedding points would be sufficient. The circular embedding of the colour example in Sect. 5 is such an application. On the contrary, the circle fitting example would need to select the best embedding points by the orthogonal Procrustes analysis, which will be described in Sect. 5 .

2.2. The Matrix Optimization Formulation. Returning to our problem, we recall that our intention was to find $n$ points $\left\{x^{1}, \ldots, x^{n}\right\}$ embedded on a sphere in $\mathbb{R}^{r}$ satisfying certain optimal criterion. The available information for us is the set of approximate (squared) Euclidean distances among the $n$ points:

$$
D_{i j}^{0} \approx\left\|x^{i}-x^{j}\right\|^{2}, \quad i, j=1, \ldots, n .
$$

Denote the center of the sphere by $x^{n+1}$ (the $(n+1)$ th point) and its radius by $R$. Since the $n$ points are placed on the sphere, we must have

$$
\left\|x^{j}-x^{n+1}\right\|=R, \quad j=1, \ldots, n .
$$

Although we do not know the exact magnitude of $R$, we can be sure that twice the radius cannot be bigger than the diameter of the data set:

$$
2 R \leq d_{\max }:=\max _{i, j} \sqrt{D_{i j}^{0}} .
$$

We therefore define the approximate distance matrix $D \in \mathcal{S}^{n+1}$ by (only upper part of $D$ is defined)

$$
D_{i j}= \begin{cases}\frac{1}{4} d_{\max }^{2} & i=1, \ldots, n, j=n+1 \\ D_{i j}^{0} & i<j=2, \ldots, n \\ 0 & i=j,\end{cases}
$$

The elements in $D$ are approximate Euclidean distances among the $(n+1)$ points $\left\{x^{1}, \ldots, x^{n+1}\right\}$. But $D$ may not be a true EDM. Our purpose is to find the nearest EDM $Y$ to $D$ such that the embedding dimension of $Y$ is $r$ and its embedding points $\left\{x^{1}, \ldots, x^{n}\right\}$ are on a sphere centered at $x^{n+1}$. The resulting matrix optimization model is then given by

$$
\begin{array}{ll}
\min _{Y \in \mathcal{S}^{n+1}} & \frac{1}{2}\|Y-D\|^{2} \\
\text { s.t. } & Y \in \mathcal{S}_{h}^{n+1},-Y \in \mathcal{K}_{+}^{n+1}, \operatorname{rank}(J Y J) \leq r \\
& Y_{1(n+1)}=Y_{j(n+1)}, \quad j=2, \ldots, n .
\end{array}
$$

We have following remarks regarding model (5).

(R1) Problem (5) is always feasible (e.g., the zero matrix is feasible). The feasible region is closed and the objective function is coercive. Let $\bar{Y}$ be its optimal solution. The first group of constraints in (5) implies that $\bar{Y}$ is an EDM with an embedding dimension not greater than $r$. If $r<n$ (i.e., $\operatorname{rank}(J Y J)<n$ ), the problem is nonconvex. If $r=n$, then we can drop the rank constraint so that the problem is convex. This is due to the fact that any EDM of size $(n+1) \times(n+1)$ has an embedding dimension not greater than $(n+1-1)=n$. Therefore, the rank constraint is automatically satisfied if $r=n$. Assume that

$$
-\frac{1}{2} J \bar{Y} J=: X^{T} X
$$


where $X \in \mathbb{R}^{r \times(n+1)}$. Let $x^{i}$ be the $i$ th column of $X$. We then have

$$
\bar{Y}_{i j}=\left\|x^{i}-x^{j}\right\|^{2}, \quad i, j=1, \ldots, n+1 .
$$

The second group of constraints in (5) means that the distances from $x^{i}$, $i=1, \ldots, n$ to $x^{n+1}$ are equal. Hence, $\left\{x^{1}, \ldots, x^{n}\right\}$ lie on a sphere centered at $x^{n+1}$. We call those constraints spherical constraints and we note that they are linear. This is in contrast to the nonlinear formulation of the spherical constraints in the previous studies $[5,28,10,13]$.

(R2) If $D^{0}$ is a true EDM from $n$ points $\left\{z^{1}, \ldots, z^{n}\right\}$ on a sphere centered at $z^{n+1}$ and let $D$ be defined by (4), then $\bar{Y}=D$ and $\left\{x^{1}, \ldots, x^{n+1}\right\}$ can be exactly matched to $\left\{z^{1}, \ldots, z^{n+1}\right\}$ through the orthogonal Procrustes analysis (see Sect. 5). If $D^{0}$ is just an approximate matrix to a true EDM on a sphere, then problem (5) finds the nearest $\operatorname{EDM} \bar{Y}$ on a sphere from $D$ in the sense that the total deviation of $D$ from the true EDM is the smallest. This optimal criterion is similar to the one used in MDS-Q [13].

(R3) The idea of introducing a variable representing the center (i.e., one more dimension in our formulation) is similar to that of [5, 28], whose main purpose was for the case $r=2$ and the variables of the optimization problems are the coordinates of the points concerned. Our model is more general for arbitrary $r$ and is conducive to (second-order) algorithmic development because the spherical constraints are linear. Furthermore, the actual embedding is left out as a separate issue, which can be done by (6), possibly through Procrustes analysis.

(R4) The following reasoning further justifies why it is necessary to introduce a new point for the center of the sphere. Let $D^{0}$ denote the true squared Euclidean distance matrix among $n$ points on a sphere. By SchoenbergYoung-Householder theorem [46, 53], the decomposition

$$
-\frac{1}{2} J D^{0} J=X^{T} X \quad \text { with } \quad J:=I_{n}-\frac{1}{n} e e^{T} \quad \text { and } \quad X \in \mathbb{R}^{r \times n},
$$

would provide a set of points $\left\{x^{i}: i=1, \ldots, n\right\}$ such that the distances in $D^{0}$ are recovered through $D_{i j}^{0}=\left\|x^{i}-x^{j}\right\|^{2}$. In order for those points to lie on a sphere centered at origin, it is necessary and sufficient to enforce the constraints

$$
\left\|x^{1}\right\|=\left\|x^{2}\right\|=\cdots=\left\|x^{n}\right\| .
$$

We note that

$$
\begin{aligned}
\left\|x^{i}\right\|^{2} & =e_{i}^{T}\left(X^{T} X\right) e_{i}=-\frac{1}{2} e_{i}^{T} J D^{0} J e_{i} \\
& =D_{i i}^{0}+\frac{1}{2 n}\left(e_{i} D^{0} e+e^{T} D^{0} e_{i}\right)-\frac{e^{T} D^{0} e}{2 n^{2}} \\
& =\frac{1}{2 n}\left\langle D^{0}, A^{i}\right\rangle-\frac{e^{T} D^{0} e}{2 n^{2}},
\end{aligned}
$$

where $A^{i}:=e_{i} e^{T}+e e_{i}^{T}$. The spherical constraints are then equivalent to

$$
\left\langle D^{0}, A^{1}-A^{i}\right\rangle=0, \quad i=2, \cdots, n,
$$


which are linear in the Euclidean distance matrix $D^{0}$. It seems that there is no need to introduce a new point to represent the center of the sphere. However, there is a potential conflict in this seemingly correct argument. We note that there is an implicit constraint we ignored. In (7), the embedding points in $X$ have to satisfy the centralization condition (because of the projection matrix $J)$

$$
X e=0 .
$$

A potential conflict is that the constraints (8) and (9) may be contradicting to each other. Such possible contradiction can be verified through the following example: Let $D^{0}$ be from the tree points on the unit circle centered at origin:

$$
x^{1}=(1,0)^{T}, \quad x^{2}=(-1,0)^{T}, \quad x^{3}=(0,1)^{T} .
$$

There exists no $X \in \mathbb{R}^{2 \times 3}$ that satisfies (7) (hence (9)) and (8). Now we define $D$ by (4) and solves problem (5), we obtain the following 4 embedding points:

$$
z^{1}=(-1,0.25)^{T}, \quad z^{2}=(1,0.25)^{T}, \quad z^{3}=(0,-0.75)^{T}, \quad z^{4}=(0,0.25)^{T} .
$$

The first three points are on the unit circle centered at $z^{4}$. The original three points $x^{1}, x^{2}$ and $x^{3}$ can be obtained through the simple shift $x^{i}=z^{i}-z^{4}$ (the simplest Procrustes analysis). This example shows that it is necessary to introduce a new point to represent the center in order to remove the potential confliction in representing the spherical constraints as linear equations.

We now reformulate (5) in a more conventional format. By replacing $Y$ by $(-Y)$ (in order to get rid of the minus sign before $\mathcal{K}_{+}^{n+1}$ ), we obtain

$$
\begin{array}{ll}
\min _{Y \in \mathcal{S}^{n+1}} & \frac{1}{2}\|Y+D\|^{2} \\
\text { s.t. } & Y \in \mathcal{S}_{h}^{n+1}, Y \in \mathcal{K}_{+}^{n+1}, \operatorname{rank}(J Y J) \leq r \\
& Y_{1(n+1)}=Y_{j(n+1)}, \quad j=2, \ldots, n .
\end{array}
$$

Define three linear mappings $\mathcal{A}_{1}: \mathcal{S}^{n+1} \mapsto \mathbb{R}^{n+1}, \mathcal{A}_{2}: \mathcal{S}^{n+1} \mapsto \mathbb{R}^{n-1}$ and $\mathcal{A}$ : $\mathcal{S}^{n+1} \mapsto \mathbb{R}^{2 n}$ respectively by

$$
\mathcal{A}_{1}(Y):=\operatorname{diag}(Y), \mathcal{A}_{2}(Y):=\left(Y_{1(n+1)}-Y_{j(n+1)}\right)_{j=2}^{n} \text { and } \mathcal{A}(Y):=\left(\begin{array}{c}
\mathcal{A}_{1}(Y) \\
\mathcal{A}_{2}(Y)
\end{array}\right) .
$$

It is therefore that solving (5) is equivalent to solving the following problem

$$
\begin{array}{ll}
\min _{Y \in \mathcal{S}^{n+1}} & \frac{1}{2}\|Y+D\|^{2} \\
\text { s.t. } & \mathcal{A}(Y)=0, \quad Y \in \mathcal{K}_{+}^{n+1} \\
& \operatorname{rank}(J Y J) \leq r .
\end{array}
$$

We note that without the spherical constraints $\mathcal{A}_{2}(Y)=0$, the problem reduces to the problem studied in Qi and Yuan [41]. However, with the spherical constraints, the analysis in [41], especially for the semismooth Newton-CG method developed in $[39,41]$ is not valid any more because it heavily depends on the simple structure of the diagonal constraints $\mathcal{A}_{1}(Y)=0$. One of our main tasks in this paper is to develop more general analysis that covers the spherical constraints. 
3. Convex Relaxation. The convex relaxation is obtained by dropping the rank constraint from (10).

$$
\begin{array}{ll}
\min _{Y \in \mathcal{S}^{n+1}} & \frac{1}{2}\|Y+D\|^{2} \\
\text { s.t. } & \mathcal{A}(Y)=0, \quad Y \in \mathcal{K}_{+}^{n+1} .
\end{array}
$$

The convex relaxation is not only important on its own right but also plays a vital role in our algorithm because a sequence of such convex problems will be solved. This section has two parts. The first part is about two constraint qualifications that the convex relaxation may enjoy. The second part is about the semismooth NewtonCG method that solves the convex relaxation and it is proved to be quadratically convergent under the qualification of constraint nondegeneracy.

3.1. Constraint Qualifications. Constraints qualifications are essential properties in deriving optimality conditions and effective algorithms for optimization problems, see, e.g., [3]. We only study two of them, which are pertinent to our numerical method to be developed later on. The first is the generalized Slater condition and the second is constraint nondegeneracy.

It follows from $[39$, p. 71$]$ that the convex cone $\mathcal{K}_{+}^{n+1}$ can be characterized as follows.

$$
\mathcal{K}_{+}^{n+1}=\left\{Q\left[\begin{array}{cc}
Z & z \\
z^{T} & z_{0}
\end{array}\right] Q: \begin{array}{l}
Z \in \mathcal{S}_{+}^{n} \\
z \in \mathbb{R}^{n}, z_{0} \in \mathbb{R}
\end{array}\right\}
$$

It is easy to see that the linear equations in $\mathcal{A}(Y)=0$ are linearly independent. We further have.

Proposition 3.1. The generalized Slater condition hold for the convex relaxation (11). That is, there exists $Y \in \mathcal{S}^{n+1}$ such that

$$
\mathcal{A}(Y)=0 \quad \text { and } \quad Y \in \operatorname{int} \mathcal{K}_{+}^{n+1},
$$

where int $\mathcal{K}_{+}^{n+1}$ denotes the interior of $\mathcal{K}_{+}^{n+1}$.

Proof. Let $x^{i}=(\sqrt{2} / 2)\left(e_{i}-(1 /(n+1)) e\right), i=1, \ldots, n+1$, where $e_{i}$ is the $i$ th unit vector in $\mathbb{R}^{n+1}$. Define $Y \in \mathcal{S}^{n+1}$ by

$$
Y_{i j}=\left\|x^{i}-x^{j}\right\|^{2}= \begin{cases}1 & \text { if } i \neq j \\ 0 & \text { if } i=j .\end{cases}
$$

It follows from Schoenberg-Young-Householder theorem $[46,53]$ that

$$
-\frac{1}{2} J Y J=\left[\begin{array}{c}
\left(x^{1}\right)^{T} \\
\vdots \\
\left(x^{n+1}\right)^{T}
\end{array}\right]\left[x^{1}, \ldots, x^{n+1}\right] \quad \text { and } \quad \operatorname{rank}(J Y J)=n .
$$

Moreover, $(-Y) \in \mathcal{K}_{+}^{n+1}$. By formula (12), there exist $Z \in \mathcal{S}_{+}^{n}, z \in \mathbb{R}^{n}$ and $z_{0} \in \mathbb{R}$ such that

$$
-Y=Q\left[\begin{array}{cc}
Z & z \\
z^{T} & z_{0}
\end{array}\right] Q
$$

By using the facts in (1), we obtain that

$$
J Y J=Q\left[\begin{array}{cc}
I_{n} & 0 \\
0 & 0
\end{array}\right] Q Y Q\left[\begin{array}{cc}
I_{n} & 0 \\
0 & 0 \\
8 &
\end{array}\right] Q=-Q\left[\begin{array}{cc}
Z & 0 \\
0 & 0
\end{array}\right] Q
$$


Since the rank of $J Y J$ is $n$ and $Z \in \mathcal{S}_{+}^{n}, Z$ must be positive definite. This proves that $Y \in \operatorname{int} \mathcal{K}_{+}^{n+1}$. Apparently, $\mathcal{A}(Y)=0$ by the definition of $Y$. Hence, the generalized Slater condition holds.

The concept of constraint nondegeneracy was first studied by Robinson [43, 44] for abstract optimization problems and has been extensively used in Bonnans and Shapiro [3] and Shapiro [48] for sensitivity analysis in optimization and variational analysis. It plays a vital role in the characterizations of strong regularity (via Clark's generalized Jacobian) in nonlinear semidefinite programming (SDP) by Sun [49]. For linear SDP, it reduces to the primal (dual) nondegeneracy of Alizadeh et al. [1], see also Chan and Sun [7] for further deep implications in SDP. It has been shown fundamental in many optimization problems, see $[40,33,39,34,26]$. Our main result is that constraint nondegeneracy holds for the convex problem (11) under a very weak condition and it further ensures that the Newton-CG method is quadratically convergent. For problem (11), constraint nondegeneracy is defined as follows (note that the problem has $2 n$ linear constraints).

DEFINITION 3.2. We say that constraint nondegeneracy holds at a feasible point $\bar{A}$ of (11) if

$$
\mathcal{A}\left(\operatorname{lin}\left(\mathcal{T}_{\mathcal{K}_{+}^{n+1}}(\bar{A})\right)\right)=\mathbb{R}^{2 n}
$$

where $\mathcal{T}_{\mathcal{K}_{+}^{n+1}}(\bar{A})$ is the tangent cone of $\mathcal{K}_{+}^{n+1}$ at $\bar{A}$ and $\operatorname{lin}\left(\mathcal{T}_{\mathcal{K}_{+}^{n+1}}(\bar{A})\right)$ is the largest subspace contained in $\mathcal{T}_{\mathcal{K}_{+}^{n+1}}(\bar{A})$.

Let $\bar{A} \in \mathcal{K}_{+}^{n+1}$ and denote

$$
\bar{A}=Q\left[\begin{array}{cc}
Z & z \\
z^{T} & z_{0}
\end{array}\right] Q, \quad Z \in \mathcal{S}_{+}^{n} .
$$

We assume that $\operatorname{rank}(Z)=r$ and let $\lambda_{1} \geq \lambda_{2} \geq \ldots \geq \lambda_{r}>0$ be the $r$ positive eigenvalues of $Z$ in nonincreasing order. Let $\Lambda:=\operatorname{Diag}\left(\lambda_{1}, \ldots, \lambda_{r}\right)$. We assume that $Z$ takes the following spectral decomposition

$$
Z=U\left[\begin{array}{ll}
\Lambda & \\
& 0
\end{array}\right] U^{T}
$$

where $U \in \mathbb{R}^{n \times n}$ and $U^{T} U=I_{n}$. Let

$$
\bar{U}:=\left[\begin{array}{cc}
U & 0 \\
0 & 1
\end{array}\right] \in \mathbb{R}^{(n+1) \times(n+1)} .
$$

Then $\bar{U}^{T} \bar{U}=I$. It follows from [39, Eq. (24)] that

$$
\left.\operatorname{lin}\left(\mathcal{T}_{\mathcal{K}_{+}^{n}}(\bar{A})\right)=\left\{Q \bar{U}\left[\left[\begin{array}{cc}
\Sigma_{1} & \Sigma_{12} \\
\Sigma_{12}^{T} & 0
\end{array}\right] \quad \begin{array}{c}
a \\
a^{T}
\end{array}\right] a_{0}\right] \bar{U}^{T} Q: \begin{array}{l}
\Sigma_{1} \in \mathcal{S}^{r} \\
\\
\Sigma_{12} \in \mathbb{R}^{r \times(n-r)} \\
a \in \mathbb{R}^{n}, a_{0} \in \mathbb{R}
\end{array}\right\} .
$$

Consider matrix $X$ of the following form:

$$
X:=Q \bar{U}\left[\begin{array}{cc}
\Gamma & -\Gamma q+\sqrt{n+1} a \\
(-\Gamma q+\sqrt{n+1} a)^{T} & q^{T} \Gamma q
\end{array}\right] \bar{U}^{T} Q
$$


where

$$
\Gamma:=\left[\begin{array}{cc}
\Sigma_{1} & \Sigma_{12} \\
\Sigma_{12}^{T} & 0
\end{array}\right] \in \mathcal{S}^{n}, \quad q:=U^{T} e, \quad a \in \mathbb{R}^{n} .
$$

Obviously, $X \in \operatorname{lin}\left(\mathcal{T}_{\mathcal{K}_{+}^{n}}(\bar{A})\right)$. Define two linear mappings $\widetilde{\mathcal{A}}_{i}: \mathcal{S}^{n} \mapsto \mathbb{R}^{n}$ for $i=1,2$ respectively by

$$
\widetilde{\mathcal{A}}_{1}(Y)=\left(Y_{11}, Y_{22}, \ldots, Y_{n n}\right)^{T}
$$

and

$$
\widetilde{\mathcal{A}}_{2}(Y)=\left(Y_{1(n+1)}-Y_{2(n+1)}, \ldots, Y_{1(n+1)}-Y_{n(n+1)}, Y_{(n+1)(n+1)}\right)^{T} .
$$

We have the following lemma.

Lemma 3.3. For any given $y \in \mathbb{R}^{n}$, there exists $a \in \mathbb{R}^{n}$, independent of the choice of $\Gamma$ in $X$ of (18), such that

$$
\widetilde{\mathcal{A}}_{2}(X)=y \text {. }
$$

Proof. Simple calculation can verify that

$$
Q e_{n+1}=-\frac{1}{\sqrt{n+1}} e \quad \text { and } \quad Q\left(e_{1}-e_{j}\right)=e_{1}-e_{j} \quad \text { for } j=1, \ldots, n .
$$

We now calculate the elements of $\widetilde{\mathcal{A}}_{2}(X)$. For $j=1, \ldots, n-1$, we have

$$
\begin{aligned}
\left(\widetilde{\mathcal{A}}_{2}(X)\right)_{j} & =\left(e_{1}-e_{j+1}\right)^{T} Q \bar{U}\left[\begin{array}{cc}
\Gamma & -\Gamma q+\sqrt{n+1} a \\
(-\Gamma q+\sqrt{n+1} a)^{T} & q^{T} \Gamma q
\end{array}\right] \bar{U}^{T} Q e_{n+1} \\
& =\left(e_{1}-e_{j+1}\right)^{T} \bar{U}\left[\begin{array}{cc}
\Gamma & -\Gamma q+\sqrt{n+1} a \\
(-\Gamma q+\sqrt{n+1} a)^{T} & q^{T} \Gamma q
\end{array}\right]\left[\begin{array}{c}
-\frac{1}{\sqrt{n+1}} q \\
-\frac{1}{\sqrt{n+1}}
\end{array}\right] \\
& =\left(e_{1}-e_{j+1}\right)^{T} \bar{U}\left[\begin{array}{c}
-a \\
-q^{T} a
\end{array}\right] \quad \text { (using (16)) } \\
& =\left(u^{j+1}-u^{1}\right)^{T} a, \quad
\end{aligned}
$$

where $u^{j}$ denotes the $j$ th column of $U^{T}$. Similarly, we can calculate the last element of $\widetilde{\mathcal{A}}_{2}(X)$ :

$$
\begin{aligned}
\left(\widetilde{\mathcal{A}}_{2}(X)\right)_{n} & =X_{(n+1)(n+1)} \\
& =\frac{1}{\sqrt{n+1}}\left[q^{T}, 1\right]\left[\begin{array}{c}
a \\
q^{T} a
\end{array}\right] \\
& =\frac{2}{\sqrt{n+1}} q^{T} a .
\end{aligned}
$$

Then, equation (20) becomes the following simultaneous equations

$$
\begin{cases}\left\langle u^{j+1}-u^{1}, a\right\rangle & =y_{j}, j=1, \ldots, n-1 \\ \left\langle U^{T} e, a\right\rangle & =\frac{\sqrt{n+1}}{2} y_{n} .\end{cases}
$$


It is easy to verify that the vectors $\left\{u^{2}-u^{1}, \ldots, u^{n}-u^{1}, U^{T} e\right\}$ are linearly independent. Hence, there exists a unique solution $a \in \mathbb{R}^{n}$ to (21) for any given $y \in \mathbb{R}^{n}$. We also note that the solution of $a$ is independent of $\Gamma$ in $X$.

Lemma 3.4. Let $\bar{A}$ be decomposed as in (14). Suppose that there exists an eigenvector $u \in \mathbb{R}^{n}$ of $Z$ corresponding to one of its positive eigenvalues such that

$$
\tau_{i}:=u_{i}+\frac{1}{\sqrt{n+1}+1} \rho \neq 0 \quad \forall i=1, \ldots, n \quad \text { with } \quad \rho:=\sum_{j=1}^{n} u_{j} .
$$

Then for any given $z \in \mathbb{R}^{n}$ and $a \in \mathbb{R}^{n}$, there exists $\Gamma$ of the type in (19) such that

$$
\widetilde{\mathcal{A}}_{1}(X)=z,
$$

where $X$ is defined by (18).

Proof. Let $a \in \mathbb{R}^{n}$ and $z \in \mathbb{R}^{n}$ be given. Define

$$
\bar{X}:=X_{1}+X_{2}+X_{3}+X_{4},
$$

with

$X_{1}=\left[\begin{array}{ll}\Gamma & 0 \\ 0 & 0\end{array}\right], X_{2}=\left[\begin{array}{cc}0 & -\Gamma q \\ -(\Gamma q)^{T} & 0\end{array}\right], X_{3}=\left[\begin{array}{cc}0 & 0 \\ 0 & q^{T} \Gamma q\end{array}\right], X_{4}=\sqrt{n+1}\left[\begin{array}{cc}0 & a \\ a^{T} & 0\end{array}\right]$.

We calculate the first $n$ diagonal elements of $X$. For $i=1, \ldots, n$, we have

$$
\begin{aligned}
X_{i i} & =e_{i}^{T} Q \bar{U}\left[\begin{array}{cc}
\Gamma & -\Gamma q+\sqrt{n+1} a \\
(-\Gamma q+\sqrt{n+1} a)^{T} & q^{T} \Gamma q
\end{array}\right] \bar{U}^{T} Q e_{i} \\
& =\left\langle\bar{U}^{T} Q e_{i} e_{i}^{T} Q \bar{U}, \bar{X}\right\rangle=\left\langle W_{i}, \bar{X}\right\rangle,
\end{aligned}
$$

where $W_{i}:=\bar{U}^{T} Q e_{i} e_{i}^{T} Q \bar{U}$. Then equation (23) becomes

$$
\left\langle W_{i}, X_{1}+X_{2}+X_{3}\right\rangle=z_{i}-\left\langle W_{i}, X_{4}\right\rangle, \quad i=1, \ldots, n .
$$

We would like to determine what $\Gamma$ satisfies (24).

Note that for $i=1, \ldots, n$,

$$
Q e_{i}=e_{i}-\frac{1}{n+1+\sqrt{n+1}} v
$$

and

$$
\begin{aligned}
W_{i} e_{n+1} & =\bar{U}^{T} Q e_{i} e_{i}^{T} Q \bar{U} e_{n+1} \\
& =\bar{U}^{T} Q e_{i} e_{i}^{T} Q e_{n+1}=-\frac{1}{\sqrt{n+1}} \bar{U}^{T} Q e_{i} \\
& =-\frac{1}{\sqrt{n+1}}\left[\begin{array}{c}
U^{T} e_{i} \\
0
\end{array}\right]+\frac{1}{(n+1)(\sqrt{n+1}+1)}\left[\begin{array}{c}
U^{T} e \\
1+\sqrt{n+1}
\end{array}\right] .
\end{aligned}
$$

We derive the following identities (we omit some details of the calculations)

$$
\begin{aligned}
\left\langle W_{i}, X_{1}\right\rangle & =\operatorname{Tr}\left(\left[U^{T}, 0\right] Q e_{i} e_{i} Q^{T}\left[\begin{array}{c}
U \\
0
\end{array}\right] \Gamma\right) \\
& =\left\langle U^{T}\left(e_{i}-\frac{1}{n+1+\sqrt{n+1}} e\right)\left(e_{i}-\frac{1}{n+1+\sqrt{n+1}} e\right)^{T} U, \Gamma\right\rangle .
\end{aligned}
$$




$$
\begin{aligned}
\left\langle W_{i}, X_{2}\right\rangle & =-2\left\langle W_{i} e_{n+1},\left[q^{T} \Gamma, 0\right]^{T}\right\rangle \\
& =\frac{2}{\sqrt{n+1}}\left(q^{T} \Gamma U^{T} e_{i}\right)-\frac{2}{(n+1)(\sqrt{n+1}+1)}\left(q^{T} \Gamma U^{T} e\right) \\
& =\frac{1}{\sqrt{n+1}}\left\langle U^{T}\left(e e_{i}^{T}+e_{i} e^{T}\right) U, \Gamma\right\rangle-\frac{2}{(n+1)(\sqrt{n+1}+1)}\left\langle U^{T} e e^{T} U, \Gamma\right\rangle .
\end{aligned}
$$

$$
\left\langle W_{i}, X_{3}\right\rangle=q^{T} \Gamma q\left(e_{n+1}^{T} Q e_{i} e_{i}^{T} Q e_{n+1}\right)=\frac{1}{n+1} q^{T} \Gamma q=\frac{1}{n+1}\left\langle U^{T} e e^{T} U, \Gamma\right\rangle .
$$

The fact $q=U^{T} e$ was used above. We add together the identities above and simplify to get

$$
\left\langle W_{i}, X_{1}+X_{2}+X_{3}\right\rangle=\left\langle U^{T} \bar{W}_{i} U, \Gamma\right\rangle
$$

with

$$
\bar{W}_{i}:=\left(e_{i}+\frac{1}{\sqrt{n+1}+1} e\right)\left(e_{i}+\frac{1}{\sqrt{n+1}+1} e\right)^{T} .
$$

Now we assume that condition (22) holds. Without loss of generality, we assume that $u$ is the leading eigenvector of $Z$ corresponding to the largest eigenvalue $\lambda_{1}$. Let $\gamma \in \mathbb{R}^{n}$ and define $\Gamma \in \mathcal{S}^{n}$ by

$$
\Gamma_{i j}:= \begin{cases}\gamma_{1} & \text { if } i=j=1 \\ \gamma_{j} / 2 & \text { if } i=1, j \geq 2 \\ \gamma_{i} / 2 & \text { if } j=1, i \geq 2 \\ 0 & \text { otherwise }\end{cases}
$$

Such $\Gamma$ is consistent with the structure in (19). It follows from (25) that

$$
\left\langle W_{i}, X_{1}+X_{2}+X_{3}\right\rangle=\left\langle U^{T} \bar{W}_{i} U e_{1}, \gamma\right\rangle=\left\langle\bar{W}_{i} U e_{1}, U \gamma\right\rangle=\left\langle\bar{W}_{i} u, \bar{\gamma}\right\rangle,
$$

where $\bar{\gamma}:=U \gamma$ and the fact $u=U e_{1}$ was used. Then the linear equations in (24) become

$$
\left\langle\bar{W}_{i} u, \bar{\gamma}\right\rangle=z_{i}-\left\langle W_{i}, X_{4}\right\rangle, \quad i=1, \ldots, n
$$

with $\bar{\gamma}$ being unknown. To ensure the existence of $\bar{\gamma}$ that satisfies (26), it is enough to prove the linear independence of the vectors $\left\{\bar{W}_{i} u\right\}_{i=1}^{n}$. Assume that there exist $\mu_{i} \in \mathbb{R}, i=1 \ldots, n$ such that

$$
\sum_{i=1}^{n} \mu_{i} \bar{W}_{i} u=0
$$

which implies (by using the structure of $\bar{W}_{i}$ )

$$
\left\{\begin{array}{lcc}
\mu_{1} \tau_{1}+\frac{1}{\sqrt{n+1}+1} \sum_{i=1}^{n} \mu_{i} \tau_{i} & = & 0 \\
\vdots & \vdots & \vdots \\
\mu_{n} \tau_{n}+\frac{1}{\sqrt{n+1}+1} \sum_{i=1}^{n} \mu_{i} \tau_{i} & = & 0 .
\end{array}\right.
$$


We must have from the above equations that

$$
\mu_{1} \tau_{1}=\mu_{2} \tau_{2}=\cdots=\mu_{n} \tau_{n}=0 .
$$

Under the assumption of (22), we have $\mu_{i}=0$ for $i=1, \ldots, n$. Hence, the vectors $\left\{\bar{W}_{i} u\right\}_{i=1}^{n}$ are linearly independent. There is a unique $\bar{\gamma}$ satisfying (26). Therefore, $\gamma=U^{T} \bar{\gamma} . \Gamma$ is well defined and the resulting $X$ defined in (18) satisfies (23). This proves the result.

Combining the two lemmas together gives our constraint nondegeneracy result.

Proposition 3.5. Let $\bar{A}$ given by (14) be a feasible point of (11). Suppose condition (22) is satisfied for $\bar{A}$. Then constraint nondegeneracy holds at $\bar{A}$.

Proof. By the definition of constraint nondegeneracy, it is sufficient to prove that for any given $x \in \mathbb{R}^{2 n}$ there exists $X \in \operatorname{lin}\left(\mathcal{T}_{\mathcal{K}_{+}^{n+1}}(\bar{A})\right)$ such that $\mathcal{A}(X)=x$. This is equivalent to existence of $X \in \operatorname{lin}\left(\mathcal{T}_{\mathcal{K}_{+}^{n+1}}(\bar{A})\right)$ such that both (20) and (23) hold simultaneously for any given $y, z \in \mathbb{R}^{n}$. Lemmas 3.3 and 3.4 just ensured this is the case. We can choose $a \in \mathbb{R}^{n}$ first in Lemma 3.3 and then choose $\Gamma$ in Lemma 3.4 to generate the matrix $X$ of (18), which satisfies (20) and (23) simultaneously for any given $y, z \in \mathbb{R}^{n}$. Hence, constraint nondegeneracy holds at $\bar{A}$ under assumption (22).

We comment as follows as pointed out by one referee. The only condition for $\bar{A}$ in Prop. 3.5 is being feasible. However, we know that $\bar{A}=0$ is feasible and constraint nondegeneracy does not hold at 0 (can be verified directly through definition). Condition (22) serves the purpose of removing such points from the consideration. The following example shows that condition (22) holds everywhere but one point.

EXAMPle 3.6. Consider the (squared) Euclidean distance matrix

$$
\bar{A}=\left[\begin{array}{cccc}
0 & 4 & 2(1-t) & 1 \\
4 & 0 & 2(1+t) & 1 \\
2(1-t) & 2(1+t) & 0 & 1 \\
1 & 1 & 1 & 0
\end{array}\right] \quad \text { and } \quad-1 \leq t \leq 1
$$

It corresponds to a triangular embedding on a unit circle with the length of one edge equal the diameter of 2 . The remaining point of the triangle moves around the circle. Hence $\operatorname{rank}(J \bar{A} J)=2$ (i.e., $r=2$ ). The corresponding matrix $Z$ is

$$
Z=\frac{1}{18}\left[\begin{array}{ccc}
37-12 t & -35 & -5+30 t \\
-35 & 37+12 t & -5-30 t \\
-5+30 t & -5-30 t & 25
\end{array}\right]
$$

It can be verified that condition (22) is satisfied for all $t$ except $t=0$.

3.2. Semismooth Newton Method. In this subsection, we develop the semismooth Newton method for the convex relaxation problem (11). The method is shown to be quadratically convergent under constraint nondegeneracy at the optimal solution. We will use this method to solve a sequence of subproblems that will appear in solving the nonconvex problem (10).

(a) Semismooth Newton Method. The Newton method is actually designed for the Lagrangian dual problem (in the form of minimization) of (11). We omit the detailed calculations that lead to the following dual problem:

$$
\min _{y \in \mathbb{R}^{2 n}} \theta(y):=\frac{1}{2}\left\|\Pi_{\mathcal{K}_{+}^{n+1}}\left(-D+\mathcal{A}^{*}(y)\right)\right\|^{2}-\frac{1}{2}\|D\|^{2},
$$


where $\mathcal{A}^{*}: \mathbb{R}^{2 n} \mapsto \mathcal{S}^{n+1}$ is the adjoint operator of $\mathcal{A}$.

We note that the linear transformations in $\mathcal{A}$ are linearly independent and that the generalized Slater condition holds for problem (11) (see Prop. 3.1). It follows from the general results [16, Prop. 2.20, Prop. 4.11] that the dual function $\theta(\cdot)$ is coercive (i.e., $\theta(y) \rightarrow \infty$ as $\|y\| \rightarrow \infty$ ). Furthermore, because $\mathcal{K}_{+}^{n+1}$ is a closed and convex cone, $\theta(\cdot)$ is convex and continuously differentiable (see [23, Chp. IV, Example 2.1.4]). Therefore, the dual problem (27) must admit an optimal solution and the first-order optimality condition is

$$
F(y):=\nabla \theta(y)=\mathcal{A}\left(\Pi_{\mathcal{K}_{+}^{n+1}}\left(-D+\mathcal{A}^{*}(y)\right)\right)=0 .
$$

It follows from the projection formula (3) of Gaffke and Mathar that $F(y)$ is strongly semismooth ${ }^{1}$ because it is a composition of linear mappings and $\Pi_{\mathcal{S}_{+}^{n+1}}(\cdot)$, which is known to be strongly semismooth [50,6]. Now it becomes natural to develop the semismooth Newton method: Given $y^{0} \in \mathbb{R}^{2 n}$ and let $k:=0$. Compute $V_{k} \in \partial F\left(y^{k}\right)$ and

$$
y^{k+1}=y^{k}-V_{k}^{-1} F\left(y^{k}\right), \quad k=0,1,2, \ldots
$$

Since $F$ is the gradient of $\theta, \partial F$ is often called the generalized Hessian of $\theta$.

According to the basic theory (see e.g. [42, Thm. 3.2]) for the semismooth Newton method, a key condition for it to be quadratically convergent is that the generalized Jacobian $\partial F\left(y^{*}\right)$ is nonsingular, where $y^{*}$ denotes the optimal solution of (27). The optimal solution $Y^{*}$ for the original convex problem (11) can be computed by

$$
Y^{*}=\Pi_{\mathcal{K}_{+}^{n+1}}\left(-D+\mathcal{A}^{*}\left(y^{*}\right)\right) .
$$

To practically implement the semismooth Newton method (29), we have to address two key issues. One is how to compute a particular matrix $V \in \partial F(y)$. This has led us to use $\widehat{\partial} F(y)$ instead (to be developed in part (c) in this subsection and also see (41)). The other issue is how to solve the linear equation in (29). Direct evaluation of $V$ would need $O\left(n^{4}\right)$ flops and hence direct methods are very expensive. We choose to use the well-developed conjugate gradient (CG) method to solve the Newton equation (see Qi and Sun [40] and Zhao et al. [54]). This results in the Newton-CG method that does not need to explicitly form the matrix $V$. The main task below is to show that the nonsingularity of $\widehat{\partial} F\left(y^{*}\right)$ (hence of $\partial F(y)$ ) under constraint nondegeneracy at $Y^{*}$.

(b) Characterization of Constraint Nondegeneracy. Let $\bar{A} \in \mathcal{K}_{+}^{n+1}$ be decomposed as in (14) and let $\lambda_{1} \geq \lambda_{2} \ldots \geq \lambda_{r}>0$ be the positive eigenvalues of $Z$ in nonincreasing order. Let $\alpha:=\{1,2, \ldots, r\}$. We have the following characterization of constraint nondegeneracy at $\bar{A}$.

Lemma 3.7. Let $h \in \mathbb{R}^{2 n}$ be given. Denote

$$
\underline{H}=\left[\begin{array}{ll}
\underline{H}_{1} & \underline{h} \\
\underline{h}^{T} & \underline{h}_{0}
\end{array}\right]:=Q\left(\mathcal{A}^{*}(h)\right) Q \quad \text { with } \underline{H}_{1} \in \mathcal{S}^{n}, \underline{h} \in \mathbb{R}^{n} \text { and } \underline{h}_{0} \in \mathbb{R} \text {. }
$$

\footnotetext{
${ }^{1} \mathrm{~A}$ (locally) Lipschitz function $\Phi: \mathbb{R}^{m} \mapsto \mathbb{R}^{\ell}$ is said to be strongly semismooth at $x \in \mathbb{R}^{m}$ if (i) $\Phi$ is directionally differentiable at $x$, and (ii) for any $V \in \partial \Phi(x+h)$,

$$
\Phi(x+h)-\Phi(x)-V h=o\left(\|h\|^{2}\right), \quad h \in \mathbb{R}^{m},
$$

where $\partial \Phi(x)$ denotes the generalized Jacobian of $\Phi$ at $x$ in the sense of Clarke [8, Sect. 2.6].
} 
Let $\bar{A} \in \mathcal{K}_{+}^{n+1}$ be decomposed as in (14) and the resulting $Z$ has the spectral decomposition (15). Constraint nondegeneracy holds at $\bar{A}$ if and only if the following implication holds

$$
\left.\begin{array}{rl}
U_{\alpha}^{T} \underline{H}_{1} & =0 \\
\underline{h} & =0 \\
\underline{h}_{0} & =0
\end{array}\right\} \Longrightarrow h=0
$$

Proof. By (13), constraint nondegeneracy holds at $\bar{A}$ if and only if

$$
h \in\left\{\mathcal{A}\left(\operatorname{lin}\left(\mathcal{T}_{\mathcal{K}_{+}^{n+1}}(\bar{A})\right)\right)\right\}^{\perp} \Longrightarrow h=0 .
$$

It follows from (17) that

$$
\left\{Q B Q: B \in \operatorname{lin}\left(\mathcal{T}_{\mathcal{K}_{+}^{n+1}}(\bar{A})\right)\right\}=\left\{\left[\begin{array}{ccc}
U\left[\begin{array}{cc}
\Sigma_{1} & \Sigma_{12} \\
\Sigma_{12}^{T} & 0
\end{array}\right] U^{T} & a \\
& a^{T} & \\
& a_{0}
\end{array}\right]: \begin{array}{l}
\Sigma_{1} \in \mathcal{S}^{r} \\
\\
\\
\Sigma_{12} \in \mathbb{R}^{r \times(n-r)} \\
a \in \mathbb{R}^{n}, a_{0} \in \mathbb{R}
\end{array}\right\} .
$$

The left-hand side of (33) is equivalent to, for any $B \in \operatorname{lin}\left(\mathcal{T}_{\mathcal{K}_{+}^{n+1}}(\bar{A})\right)$,

$$
\begin{aligned}
0 & =\langle h, \mathcal{A}(B)\rangle=\left\langle\mathcal{A}^{*}(h), B\right\rangle \\
& \left.=\left\langle Q \mathcal{A}^{*}(h) Q, Q B Q\right\rangle \quad \quad \text { (because } Q^{2}=I\right) \\
& =2\langle\underline{h}, a\rangle+\underline{h}_{0} a_{0}+\operatorname{Tr}\left(U^{T} \underline{H}_{1} U\left[\begin{array}{cc}
\Sigma_{1} & \Sigma_{12} \\
\Sigma_{12}^{T} & 0
\end{array}\right]\right) .
\end{aligned}
$$

The above identities are for any $a \in \mathbb{R}^{n}, a_{0} \in \mathbb{R}, \Sigma_{1} \in \mathcal{S}^{r}$ and $\Sigma_{12} \in \mathbb{R}^{r \times(n-r)}$. Hence, we must have (recall $\alpha=\{1,2, \ldots, r\}$ )

$$
\underline{h}=0, \quad \underline{h}_{0}=0 \quad \text { and } \quad U_{\alpha}^{T} \underline{H}_{1} U=0 .
$$

Because of the nonsingularity of $U$, the above condition is equivalent to

$$
\underline{h}=0, \quad \underline{h}_{0}=0 \quad \text { and } \quad U_{\alpha}^{T} \underline{H}_{1}=0 .
$$

Therefore, (33) holds if and only if (32) holds.

(c) Structure of $\widehat{\partial} F(y)$. For a given $y \in \mathbb{R}^{2 n}$, we let

$$
Y:=-J\left(-D+\mathcal{A}^{*}(y)\right) J \quad \text { and } \quad \bar{A}:=\Pi_{\mathcal{K}_{+}^{n+1}}\left(-D+\mathcal{A}^{*}(y)\right) .
$$

Denote

$$
\left[\begin{array}{cc}
\underline{Z} & \underline{z} \\
\underline{z}^{T} & \underline{z}_{0}
\end{array}\right]:=-Q\left(-D+\mathcal{A}^{*}(y)\right) Q \quad \text { with } \underline{Z} \in \mathcal{S}^{n}, \underline{z} \in \mathbb{R}^{n}, \underline{z}_{0} \in \mathbb{R} .
$$

We then have from (1) that

$$
Y=Q\left[\begin{array}{cc}
\underline{Z} & 0 \\
0 & 0
\end{array}\right] Q \quad \text { and } \quad \Pi_{\mathcal{S}_{+}^{n+1}}(Y)=Q\left[\begin{array}{cc}
\Pi_{\mathcal{S}_{+}^{n}}(\underline{Z}) & 0 \\
0 & 0
\end{array}\right] Q
$$


We further have

$$
\begin{aligned}
Q \bar{A} Q & =Q \Pi_{\mathcal{K}_{+}^{n+1}}\left(-D+\mathcal{A}^{*}(y)\right) Q \\
& =Q\left(-D+\mathcal{A}^{*}(y)\right) Q+Q \Pi_{\mathcal{S}_{+}^{n+1}}(Y) Q \quad(\text { by }(3)) \\
& =-\left[\begin{array}{cc}
\frac{Z}{\underline{z}} & \underline{z} \\
\underline{z}^{T} & \underline{z}_{0}
\end{array}\right]+\left[\begin{array}{cc}
\Pi_{\mathcal{S}_{+}^{n}}(\underline{Z}) & 0 \\
0 & 0
\end{array}\right] \\
& =\left[\begin{array}{cc}
\Pi_{\mathcal{S}_{+}^{n}}(-\underline{Z}) & -\underline{z}^{T} \\
-\underline{z}^{T} & -\underline{z}_{0}
\end{array}\right] .
\end{aligned}
$$

We write $\bar{A}$ as in (14). It follows that

$$
Z=\Pi_{\mathcal{S}_{+}^{n}}(-\underline{Z}), \quad z=-\underline{z} \quad \text { and } \quad z_{0}=-\underline{z}_{0} .
$$

Let $\underline{Z}$ admit the following spectral decomposition

$$
\underline{Z}=W \underline{\Lambda} W^{T},
$$

with $\underline{\Lambda}:=\operatorname{Diag}\left(\underline{\lambda}_{1}, \ldots, \underline{\lambda_{n}}\right)$ and $\underline{\lambda}_{1} \geq \ldots \geq \underline{\lambda}_{n}$ being the eigenvalues of $\underline{Z}$ and $W W^{T}=$ $I_{n}$.

Define

$$
\underline{\alpha}:=\left\{i: \underline{\lambda}_{i}>0\right\}, \underline{\beta}:=\left\{i: \underline{\lambda}_{i}=0\right\} \quad \text { and } \quad \underline{\gamma}:=\left\{i: \underline{\lambda}_{i}<0\right\} .
$$

The relationship between $Y$ and $\underline{Z}$ in (34) means that $\left\{\underline{\lambda}_{1}, \ldots, \underline{\lambda}_{n}\right\}$ are the eigenvalues of $Y$. Moreover, $Y$ has just one more eigenvalue, which is zero, than $\underline{Z}$. For those eigenvalues, define the corresponding symmetric matrix $\Omega \in \mathcal{S}^{n}$ with entries

$$
\Omega_{i j}:=\frac{\max \left\{\underline{\lambda}_{i}, 0\right\}+\max \left\{\underline{\lambda}_{j}, 0\right\}}{\left|\underline{\lambda}_{i}\right|+\left|\underline{\lambda}_{j}\right|}, \quad i, j=1, \ldots, n
$$

where $0 / 0$ is defined to be 1 . Let

$$
W=\left[W_{\underline{\alpha}}, W_{\underline{\beta}}, W_{\underline{\gamma}}\right] \text { and } \bar{W}:=\left[\begin{array}{cccc}
W_{\underline{\alpha}} & W_{\underline{\beta}} & 0 & W_{\underline{\gamma}} \\
0 & 0 & 1 & 0
\end{array}\right] .
$$

It follows from (28) and (3) that

$$
F(y)=\mathcal{A}\left(-D+\mathcal{A}^{*}(y)\right)+\mathcal{A}\left(\Pi_{\mathcal{S}_{+}^{n+1}}\left(-J\left(-D+\mathcal{A}^{*}(y)\right) J\right)\right) .
$$

The key part in $F(y)$ is the composite function between $\Pi_{\mathcal{S}_{+}^{n+1}}(\cdot)$ and the linear operator $J \mathcal{A}^{*}(\cdot) J$. Because of this feature, it is hard to express $\partial F(y)$ exactly. We therefore define the following alternative:

$$
\widehat{\partial} F(y):=\mathcal{A A}^{*}(y)-\mathcal{A}\left(\partial \Pi_{\mathcal{S}_{+}^{n+1}}(Y)\left(J \mathcal{A}^{*}(\cdot) J\right)\right) .
$$

Although we do not know whether $\partial F(y)$ is contained in $\widehat{\partial} F(y)$, their images of vectors coincide:

$$
\partial F(y) h=\widehat{\partial} F(y) h, \quad \forall h \in \mathbb{R}^{2 n}
$$


which implies that if all elements in $\widehat{\partial} F(y)$ are positive definite, so are those in $\partial F(y)$. The relationship in (39) holds because $F(\cdot)$ is the gradient of a convex function and this fact implies the equivalence by [24].

The valuable benefit in using $\widehat{\partial} F(y)$ is that the set can be completely characterized because the generalized Jacobian $\partial \Pi_{\mathcal{S}_{+}^{n+1}}(\cdot)$ has a full characterization (see [49, Prop. 2.2]). We describe $\widehat{\partial} F(y)$ in the following result by making use of [49, Prop. 2.2]. Its proof can be patterned after those in Sect. 3.1 to Sect. 3.3 of [39] for [39, Prop. 3.2]. We note that only the submatrix $\Omega_{\underline{\alpha} \gamma}$ is used in the description.

Proposition 3.8. For every matrix $M \in \widehat{\partial} F(y)$, there exists $\widetilde{V} \in \partial \Pi_{\mathcal{S}_{+}^{|\underline{\beta}|+1}}(0)$ such that

$$
M h=\mathcal{A}\left(\mathcal{A}^{*}(h)\right)-\mathcal{A}\left(P \mathcal{W}_{h} P^{T}\right), \quad \forall h \in \mathbb{R}^{2 n}
$$

where $P:=Q \bar{W}$,

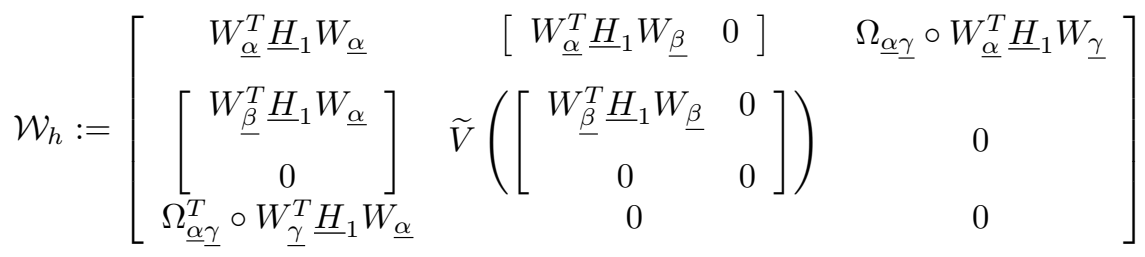

and $\underline{H}_{1}$ is from the partition in (31).

An implementable version of the semismooth Newton method (29) takes the following form

$$
y^{k+1}=y^{k}-M_{k}^{-1} F\left(y^{k}\right), \quad M_{k} \in \widehat{\partial} F\left(y^{k}\right), \quad k=0,1,2, \ldots
$$

To implement the above method, we need to choose an explicit element $M_{k} \in \widehat{\partial} F\left(y^{k}\right)$. The matrix $M$ (subscript $k$ is omitted) used in our implementation is given by (40) with $\widetilde{V}=0$. This can be proved by using Prop. 3.8 and [37, Lemma 11].

(d) Nonsingularity of $\widehat{\partial} F(y)$. Recall the matrices $\underline{H}$ and $\bar{W}$ are respectively defined in (31) and (37). It is easy to verify that

$$
\bar{W}^{T} \underline{H} \bar{W}=\left[\begin{array}{cccc}
W_{\underline{\alpha}}^{T} \underline{H}_{1} W_{\underline{\alpha}} & W_{\underline{\alpha}}^{T} \underline{H}_{1} W_{\underline{\beta}} & W_{\underline{\alpha}}^{T} \underline{h} & W_{\underline{\alpha}}^{T} \underline{H}_{1} W_{\underline{\gamma}} \\
W_{\underline{\beta}}^{T} \underline{H}_{1} W_{\underline{\alpha}} & W_{\underline{\beta}}^{T} \underline{H}_{1} W_{\underline{\beta}} & W_{\underline{\beta}}^{T} \underline{h} & W_{\underline{\beta}}^{T} \underline{H}_{1} W_{\underline{\gamma}} \\
\underline{h}^{T} W_{\underline{\alpha}} & \underline{\underline{h}^{T}} W_{\underline{\beta}} & \underline{h_{0}} & \underline{h}^{T} W_{\underline{\gamma}} \\
W_{\underline{\gamma}}^{T} \underline{H}_{1} W_{\underline{\alpha}} & W_{\underline{\gamma}}^{T} \underline{H}_{1} W_{\underline{\beta}} & W_{\underline{\gamma}}^{T} \underline{h} & W_{\underline{\gamma}}^{T} \underline{H}_{1} W_{\underline{\gamma}}
\end{array}\right] .
$$

We further denote

$$
G_{1}:=\left[\begin{array}{cc}
W_{\underline{\beta}}^{T} \underline{H}_{1} W_{\underline{\beta}} & W_{\underline{\beta}}^{T} \underline{h} \\
\underline{h}^{T} W_{\underline{\beta}} & \underline{\underline{h}}_{0}
\end{array}\right], \quad G_{2}:=\left[\begin{array}{cc}
W_{\underline{\beta}}^{T} \underline{H}_{1} W_{\underline{\beta}} & 0 \\
0 & 0
\end{array}\right] .
$$

It is easy to prove that

$$
\left\|G_{1}\right\|\left(\left\|G_{1}\right\|-\left\|G_{2}\right\|\right) \geq\left\|W_{\underline{\beta}}^{T} \underline{h}\right\|^{2}+\frac{1}{2} \underline{h}_{0}^{2}
$$


It follows from [7, Eq (17)] that

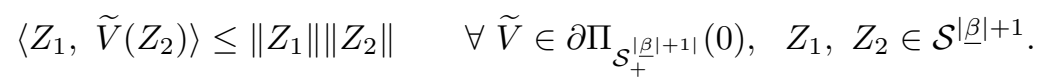

From (35), the positive eigenvalues of $Z$ are just the opposite of those negative eigenvalues in $\underline{\gamma}$. Let $\Gamma$ be the permutation matrix which maps the sequence $\{1,2, \ldots,|\underline{\gamma}|\}$ to its reverse order. We have,

$$
Z=W_{\underline{\gamma}}\left(-\Lambda_{\underline{\gamma}}\right) W_{\underline{\gamma}}=\left(W_{\underline{\gamma}} \Gamma\right)\left(\Gamma\left(-\Lambda_{\underline{\gamma}}\right) \Gamma\right)\left(W_{\underline{\gamma}} \Gamma\right)^{T} .
$$

Hence, $U_{\alpha}$, which consists of the eigenvectors of positive eigenvalues in the spectral decomposition (15), can be chosen to be

$$
U_{\alpha}=W_{\underline{\gamma}} \Gamma .
$$

TheOREm 3.9. Let $y$ be the optimal solution of the dual problem (27). Let $\bar{A}:=\Pi_{\mathcal{K}_{+}^{n+1}}\left(-D+\mathcal{A}^{*}(y)\right)$. We assume that constraint nondegeneracy holds at $\bar{A}$. Then every matrix $M \in \widehat{\partial} F(y)$ is positive definite.

Proof. We continue to use the notation developed so far. Let $M \in \widehat{\partial} F(y)$. Our purpose is to prove $\langle h, M h\rangle>0$ for all $0 \neq h \in \mathbb{R}^{2 n}$. It follows from (40)

$$
M h=\mathcal{A}\left(\mathcal{A}^{*}(h)\right)-\mathcal{A}\left(P \mathcal{W}_{h} P^{T}\right),
$$

where $\mathcal{W}_{h}$ is given in Prop. 3.8.

We now calculate $\langle h, M h\rangle$.

$$
\begin{array}{rlr}
\langle h, M h\rangle= & \left\|\mathcal{A}^{*}(h)\right\|^{2}-\left\langle\mathcal{A}^{*}(h), P \mathcal{W}_{h} P^{T}\right\rangle=\left\|Q \mathcal{A}^{*}(h) Q\right\|^{2}-\left\langle P^{T} \mathcal{A}^{*}(h) P, \mathcal{W}_{h}\right\rangle \\
= & \|\underline{H}\|^{2}-\left\langle\bar{W}^{T} \underline{H} \bar{W}, \mathcal{W}_{h}\right\rangle & (\text { by }(31) \text { and } P=Q \bar{W}) \\
= & \left\|\bar{W}^{T} \underline{H} \bar{W}\right\|^{2}-\left\langle\bar{W} \underline{H} \underline{W}, \mathcal{W}_{h}\right\rangle & \left(\text { by } \bar{W} \bar{W}^{T}=I_{n+1}\right) \\
= & 2\left\{\left\|W_{\underline{\alpha}}^{T} \underline{h}\right\|^{2}+\left\|W_{\underline{\alpha}}^{T} \underline{H}_{1} W_{\underline{\gamma}}\right\|^{2}-\left\langle W_{\underline{\alpha}}^{T} \underline{H}_{1} W_{\underline{\gamma}}, \Omega_{\underline{\alpha} \underline{\gamma}} \circ\left(W_{\underline{\alpha}}^{T} \underline{H}_{1} W_{\underline{\gamma}}\right)\right\rangle\right\} \\
& +2\left\{\left\|W_{\underline{\beta}}^{T} \underline{H}_{1} W_{\underline{\gamma}}\right\|^{2}+\left\|W_{\underline{\gamma}}^{T}\right\|^{2}+\left\|W_{\underline{\gamma}}^{T} \underline{H}_{1} W_{\underline{\gamma}}\right\|^{2} / 2\right\} \\
& +\left\|G_{1}\right\|^{2}-\left\langle G_{1}, \widetilde{V}\left(G_{2}\right)\right\rangle .
\end{array}
$$

The last equality made use of the structure of $\mathcal{W}_{h}$ and (42).

Define $\tau_{\max }:=\max _{i \in \underline{\alpha}, j \in \underline{\gamma}} \Omega_{i j}$. By (36), $0<\tau_{\max }<1$. We continue to simplify $\langle h, M h\rangle$.

$$
\begin{aligned}
\langle h, M h\rangle \geq & 2\left\{\left\|W_{\underline{\alpha}}^{T} \underline{h}\right\|^{2}+\left\|W_{\underline{\underline{\gamma}}}^{T} \underline{h}\right\|^{2}+\left\|W_{\underline{\beta}}^{T} \underline{H}_{1} W_{\underline{\gamma}}\right\|^{2}+\left(1-\tau_{\max }\right)\left\|W_{\underline{\underline{\alpha}}} \underline{H}_{1} W_{\underline{\underline{\gamma}}}\right\|^{2}\right\} \\
& +\left\|W_{\underline{\underline{\gamma}}}^{T} \underline{H}_{1} W_{\underline{\underline{\gamma}}}\right\|^{2}+\left\|G_{1}\right\|^{2}-\left\|G_{1}\right\|\left\|G_{2}\right\| \\
\geq & 2\left\{\left\|W_{\underline{\alpha}}^{T} \underline{h}\right\|^{2}+\left\|W_{\underline{\underline{\gamma}}}^{T} \underline{h}\right\|^{2}+\frac{1}{2}\left\|W_{\underline{\beta}}^{T} \underline{h}\right\|^{2}\right\}+\left\|W_{\underline{\underline{\gamma}}}^{T} \underline{H}_{1} W_{\underline{\underline{\gamma}}}\right\|^{2} \\
& +2\left\{\left(1-\tau_{\max }\right)\left\|W_{\underline{\underline{\alpha}}}^{T} \underline{H}_{1} W_{\underline{\underline{\gamma}}}\right\|^{2}+\left\|W_{\underline{\underline{\beta}}}^{T} \underline{H}_{1} W_{\underline{\gamma}}\right\|^{2}\right\}+\frac{1}{2} \underline{h}_{0}^{2} \quad \text { (by (43)) } \\
\geq & 0 .
\end{aligned}
$$

Hence, the assumption $\langle h, M h\rangle=0$ would imply

$$
W_{\underline{\alpha}}^{T} \underline{h}=0, \quad W_{\underline{\beta}}^{T} \underline{h}=0, \quad W_{\underline{\gamma}}^{T} \underline{h}=0, \quad \text { and } \quad \underline{h}_{0}=0,
$$


and

$$
W_{\underline{\underline{\alpha}}}^{T} \underline{H}_{1} W_{\underline{\underline{\gamma}}}=0, \quad W_{\underline{\beta}}^{T} \underline{H}_{1} W_{\underline{\gamma}}=0, \quad W_{\underline{\gamma}}^{T} \underline{H}_{1} W_{\underline{\gamma}}=0 .
$$

Because of (37) and nonsingularity of $W$, the two equations above yield:

$$
\underline{h}=0, \quad \underline{h}_{0}=0 \quad \text { and } \quad \underline{H}_{1} W_{\underline{\gamma}}=0,
$$

which by (45) and the nonsingularity of $\Gamma$ ( $\Gamma$ is a permutation matrix) leads to

$$
\underline{h}=0, \quad \underline{h}_{0}=0 \quad \text { and } \quad \underline{H}_{1} U_{\alpha}=0 \text {. }
$$

By assumption, constraint nondegeneracy holds at $\bar{A}$. Lemma 3.7 forces $h=0$. The inequality (46) implies that $\langle h, M h\rangle>0$ for any $h \neq 0$. Therefore, any matrix in $\widehat{\partial} F(y)$ is positive definite under constraint nondegeneracy.

(e) Quadratic convergence. The direct consequence of Thm. 3.9 is the quadratic convergence of the Newton method (41). Let $y^{*}$ be an optimal solution of the Lagrangian dual problem (27).

THEOREM 3.10. The Newton method (41) is quadratically convergent provided that $y^{0}$ is sufficiently close to the optimal solution $y^{*}$ of (27) and constraint nondegeneracy holds at $Y^{*}$ that is defined by (30).

Proof. In the general quadratic convergence-rate theorem of Qi and Sun [42, Thm. 3.2] for semismooth Newton methods, there are three conditions: (i) The function $F$ is strongly semismooth, which is true for our case because it is a composition of linear mappings and the strongly semismooth mapping $\Pi_{\mathcal{S}_{+}^{n+1}}(\cdot)$. (ii) Every matrix in the generalized Jacobian of $\widehat{\partial} F\left(y^{*}\right)$ is nonsingular, which has been proved in Thm. 3.9 under constraint nondegeneracy assumption. Furthermore, $\widehat{\partial} F(\cdot)$ is compact and upper semicontinuous. The last condition is that the initial point $y^{0}$ stays close to $y^{*}$. This proves our result.

Since (27) is convex, globalization of the Newton method (41) is straightforward. We simply use one of the well-developed globalization method (Newton-CG method) studied by Qi and Sun [40] in our numerical experiment.

4. Majorized Penalty Method. In this section, we extend the majorized penalty method of Gao and Sun [17] to our problem (10). The method has previously been used to compute the nearest EDM of low embedding dimensions in [41]. The situation here is that we have spherical constraints to deal with. The structure of the extension is similar to that in [41]. We give a brief description of the method.

(a) The penalty problem. It has been shown that without the rank constraint $\operatorname{rank}(J Y J) \leq r$, the convex relaxation problem (11) can be solved by the Newton-CG method (41). Problem (11) implicitly implies a very important fact that the matrix $(J Y J)$ is positive semidefinite for any feasible point $Y$. Define

$$
\begin{aligned}
p(Y) & :=\sum_{i=r+1}^{n} \lambda_{i}(J Y J)=\left\langle I_{n+1}, J Y J\right\rangle-\sum_{i=1}^{r} \lambda_{i}(J Y J) \\
& \left.=\langle J, Y\rangle-\sum_{i=1}^{r} \lambda_{i}(J Y J), \quad \quad \text { (because } J^{2}=J\right)
\end{aligned}
$$


where $\lambda_{1}(J Y J) \geq \ldots \geq \lambda_{n+1}(J Y J)$ are the eigenvalues of $(J Y J)$. The equivalent relationship below is obvious.

$$
\operatorname{rank}(J Y J) \leq r \quad \text { and } \quad J Y J \succeq 0 \quad \Longleftrightarrow \quad p(Y)=0 \quad \text { and } \quad J Y J \succeq 0 .
$$

Moreover, $p(Y) \geq 0$ for any $Y$ satisfying $J Y J \succeq 0$. Therefore, the function $p(Y)$ can be used as a penalty function for the rank constraint over the feasible region of (10). A similar fact has been used by Gao and Sun [17] in their majorized penalty method for computing the nearest low-rank correlation matrix, which is necessarily positive semidefinite. The resulting penalty problem in our case is

$$
\begin{array}{ll}
\min & f_{c}(Y):=f(Y)+c p(Y) \\
\text { s.t. } & \mathcal{A}(Y)=0, \quad Y \in \mathcal{K}_{+}^{n+1},
\end{array}
$$

where $c>0$ is the penalty parameter and $f(Y):=\|Y+D\|^{2} / 2$.

The following result on the relationship between the original problem (10) and its penalty counterpart (48) can be similarly proved as for [17, Prop. 3.1 and Prop. 3.2].

Proposition 4.1. Let $Y_{c}^{*}$ denote a global optimal solution of (48), $Y_{r}$ be a feasible solution of (10), and $Y^{*}$ be an optimal solution of the convex problem (11).

(i) If $\operatorname{rank}\left(Y_{c}^{*}\right) \leq r$, then $Y_{c}^{*}$ already solves (10).

(ii) If the penalty parameter $c$ is chosen to satisfy $c \geq\left(f\left(Y_{r}\right)-f\left(Y^{*}\right)\right) / \epsilon$, for some given $\epsilon>0$, then we have

$$
p\left(Y_{c}^{*}\right) \leq \epsilon \quad \text { and } \quad f\left(Y_{c}^{*}\right) \leq \nu_{*}-c p\left(Y_{c}^{*}\right),
$$

where $\nu_{*}$ denotes the optimal objective vale of (10).

The result in (ii) means that when the rank error measured by $p(\cdot)$ at $Y_{c}^{*}$ is less than $\epsilon$, the corresponding objective value comes very close to the optimal value $\nu_{*}$. Such a solution is referred to as an $\epsilon$-optimal solution in [17].

(b) Majorized Penalty Approach. The focus now is on solving the penalty problem (48). Since $p(Y)$ is concave (i.e., the sum of the first $r$ largest eigenvalues of a symmetric matrix is a convex function of the matrix), it can be majorized by the linear function defined by its subgradient: For given $Y^{k} \in \mathcal{S}^{n+1}$ (the current iterate) and $U^{k} \in \partial p\left(Y^{k}\right)$, we have

$$
p(Y) \leq m_{k}^{p}(Y):=p\left(Y^{k}\right)+\left\langle U^{k}, Y-Y^{k}\right\rangle \quad \forall Y .
$$

The function $m_{k}^{p}(Y)$ is called a majorization of $p(Y)$ at $Y^{k}$ because of (49) and $p\left(Y^{k}\right)=m_{k}^{p}\left(Y^{k}\right)$. The majorized (convex) subproblem to be solved is

$$
\min f(Y)+c m_{k}^{p}(Y), \quad \text { s.t. } \mathcal{A}(Y)=0, \quad Y \in \mathcal{K}_{+}^{n+1} .
$$

We now extend the majorized penalty algorithm of Gao and Sun [17] to our problem (10).

Algorithm 4.2. (Majorized Penalty Algorithm (MPA))

(S.1) Choose a feasible point $Y^{0}$ of (11). Set $k:=0$.

(S.2) Solve subproblem (50) to get $Y^{k+1}$.

(S.3) If $Y^{k+1}=Y^{k}$, stop; otherwise, set $k:=k+1$ and go to (S.2).

We have the following remarks about the algorithm.

(R1) There are a few choices for the starting (feasible) point $Y^{0}$ in (S.1). One of them is the optimal solution of (11) by the Newton-CG method (41). 
(R2) Alg. 4.2 generates a sequence of decreasing objective values $\left\{f_{c}\left(Y^{k}\right)\right\}$ for fixed $c$. This is because of the majorization property (49). In our practical implementation, the penalty parameter is updated according to some rules.

(R3) Subproblem (50) can be solved by the Newton-CG method (41) because it is equivalent to the following problem, which is the type of the convex problem (11) but with different input $D$ :

$$
\min \frac{1}{2}\|Y+\bar{D}\|^{2}, \quad \text { s.t. } \mathcal{A}(Y)=0, \quad Y \in \mathcal{K}_{+}^{n+1},
$$

where $\bar{D}:=D+c U^{k}$. We note that the feasible region remains unchanged. Hence, the generalized Slater condition and the constraint nondegeneracy results studied before hold for those subproblems.

(R4) The algorithm converges to a $B$-stationary point of (10), which is stated below and whose proof can be patterned after [17, Thm. 3.4]. For the definition of $B$-stationary point, see [17]. Roughly speaking, as problem (10) is nonconvex, converging to a $B$-stationary point is one kind of global convergence that the algorithm can best achieve. We omit the details. We also note that if $Y^{f}$ is the final iterate of Alg. 4.2, then $\left(-Y^{f}\right)$ should be the final output as it is a true Euclidean distance matrix (put the minus back because we have introduced the minus sign in the formulation process that led to problem $(10))$.

Proposition 4.3. Let $\left\{Y^{k}\right\}$ be the sequence generated by Alg. 4.2. Then $\left\{f_{c}\left(Y^{k}\right)\right\}$ is a monotonically decreasing sequence. If $Y^{k+1}=Y^{k}$ for some $Y^{k}$, then $Y^{k}$ is an optimal solution of (48). Otherwise, the infinite sequence $\left\{Y^{k}\right\}$ satisfies

$$
\frac{1}{2}\left\|Y^{k+1}-Y^{k}\right\|^{2} \leq f_{c}\left(Y^{k}\right)-f_{c}\left(Y^{k+1}\right), \quad k=0,1, \ldots
$$

Moreover, the sequence $\left\{Y^{k}\right\}$ is bounded and any accumulation point is a B-stationary point of (48).

5. Numerical Examples. In this section, we first briefly describe the Matlab implementation of Alg. 4.2. For ease of reference, we call the resulting code FITS ${ }^{2}$, standing for "FIT data on a Sphere". We then test a few well-known examples that have spherical constraints. Through those examples, it is demonstrated that FITS can provide data visualization of high quality and is capable of including extra (linear) constraints such as the "pole constraints" in Ekman's color example [14], which results in a wheel representation of 14 colors. We are not aware any existing methods that can deal with such pole constraints. It also provides an alternative method for the circle fitting problem, recently studied by Beck and Pan [2].

(a) Termination Criterion. We terminate Alg. 4.2 when the following two conditions are met. The first condition is on the objective function value:

$$
f_{\text {frog }}:=\frac{\left|\sqrt{f\left(Y^{k}\right)}-\sqrt{f\left(Y^{k-1}\right)}\right|}{\max \left\{100, \sqrt{f\left(Y^{k-1}\right)}\right\}} \leq \text { tol },
$$

where $f(Y)=0.5\|Y+D\|^{2}$ and tol is a small tolerance level (e.g., $1.0 \times 10^{-4}$ ). In other words, whenever there is lack of the relative progress on the successive objective

\footnotetext{
${ }^{2}$ Available from http://personal.soton.ac.uk/hdqi.
} 
function values, we believe that the current iterate is a good candidate subject to the second condition below. This stopping criterion was suggested by Gao and Sun [17] for the low-rank nearest correlation matrix problem.

The second condition is on the rank of the current iterate $Y^{k}$. There are two ways to monitor the rank. One is to compute the absolute value of the eigenvalue residue:

$$
\text { rankerror }:=\sum_{i=r+1}^{n} \lambda_{i}\left(J Y^{k} J\right) \leq \text { ranktol, }
$$

where ranktol is a small tolerance (e.g., $10^{-2}$ ) and $\lambda_{1} \geq \ldots \geq \lambda_{n}$ are the eigenvalues of $\left(J Y^{k} J\right)$, which is positive semidefinite. This quantity does not scale well with the magnitude of $\left(J Y^{k} J\right)$. To rectify this drawback, we also calculate the percentage of the first $r$ eigenvalues of $\left(J Y^{k} J\right)$ out of all the eigenvalues:

$$
\text { Eigenratio }:=\sum_{i=1}^{r} \lambda_{i}\left(J Y^{k} J\right) / \sum_{i=1}^{n} \lambda_{i}\left(J Y^{k} J\right) \geq \text { Eigentol, }
$$

where Eigentol is a high percentage (e.g., 90\%). We terminate the algorithm when (52) and either of (53) and (54) are satisfied.

(b) Initial Point and Updating the Penalty Parameter. The initial point is computed by the Semismooth Newton-CG method for the convex problem (11). We note that all the subproblems of (51) are solved by the same Newton-CG method. Alg. 4.2 solves the penalty problem (48) for a fixed penalty parameter $c$. In practical implementation, we may start from $c_{0}$ and increase $c$ a few times before we can find a good solution. The initial $c_{0}=10$ in our implementation. We update $c_{k}(k \geq 1)$ as follows

$$
c_{k}:= \begin{cases}c_{k-1}, & \text { if } \operatorname{rank}\left(J Y^{k-1} J\right) \leq r \\ 4 c_{k-1}, & \text { otherwise. }\end{cases}
$$

That is, we keep the penalty parameter unchanged if the current iterate has the desired embedding dimension. Otherwise, it is increased by 4 times.

(c) Numerical Examples. Four existing examples were tested. They are (E1) Ekman's color example [14], (E2) Trading globe in [10], (E3) 3D Map of global cities $\left(\right.$ HA30 data set ${ }^{3}$ ) in [22], and (E4) Circle fitting problem in [2]. We also show how a Procrustes procedure can be devised to assess the quality of the final embedding in (E3) or to help to get the final embedding to match the existing points in (E4).

(E1) Ekman color example. This is a classical example in MDS where data can be represented on a circle, called circular fitting. Ekman [14] presents similarities for 14 colors (wavelengths from 434 to $674 \mathrm{~nm}$ ). The similarities are based on a rating by 31 subjects where each pair of colors was rated on a 5 -point scale $(0$ means no similarity up to 4 meaning identical). After averaging, the similarities were divided by 4 such that they are within the unit interval. The similarity matrix is denoted by $\Delta$. The initial distance matrix is obtained from $\left(D^{0}\right)_{i j}:=\left(1-\Delta_{i j}\right)^{2}$.

Fig. 5.1 (a) (the radius is $R=0.5354$ ) is the resulting circular representation by FITS with colors appearing on the circle one by one in order of their wavelength.

\footnotetext{
${ }^{3}$ Data available from http://people.sc.fsu.edu/ jburkardt/m_src/distance_to_position_sphere.html
} 


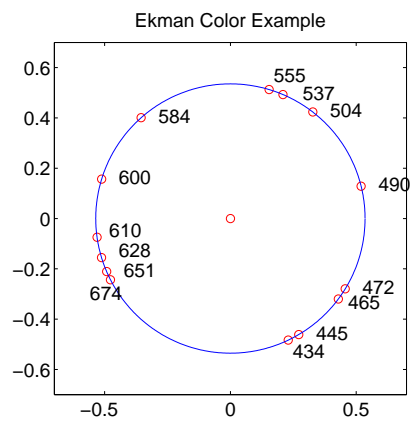

(a) Circular fitting without any constraints

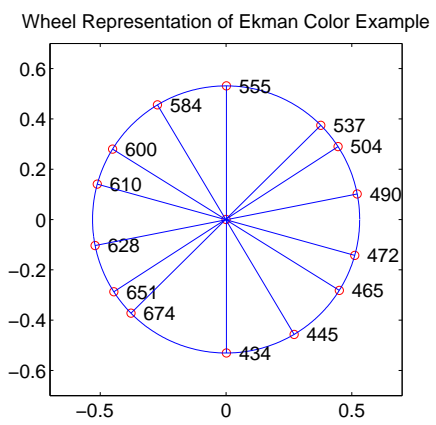

(b) Circuclar fitting with pole constraints

FIG. 5.1. Comparison between the two circular fitting of Ekman's 14 color problem with and without pole constraints.

This figure is similar to [13, Fig. 2], where more comments on this example can be found. A pair of colors $(i, j)$ are said opposing to each other if their distance equals the diameter of the circle. That is

$$
Y_{i j}=4 Y_{1(n+1)},
$$

which means that the squared distance between opposing colors is fourfold of the radius squared. This type of constraints is called "pole constraint". An interesting feature is that we assume that the first 7 colors are set to oppose the remaining 7 colors, the resulting circular representation appears as a nice wheel, without having changed the order of the colors, see Fig. 5.1(b) (the radius is 0.5310). Practitioners in Psychology may have new interpretation of such nice representation. We emphasize that our method can easily include the pole constraints and other linear constraints without any technical difficulties. We are not aware any existing methods that can directly handle those extra constraints.

(E2) Trading globe. The data in this example was first mapped to a sphere $(r=3)$ in [10] and was recently tested in [13]. The data was originally taken from the New Geographical Digest (1986) on which countries traded with other countries. For 20 countries the main trading partners are dichotomously scored ( 1 means trade performed, 0 trade not performed). Based on this dichotomous matrix $X$ the distance matrix $D^{0}$ is computed using the squared Jaccard coefficient (computed by the Matlab build-in function pdist(X, 'jaccard'). The most intuitive MDS approach is to project the resulting distances to a sphere which gives a "trading globe".

In Fig. $5.2(R=0.5428)$, the counties were projected on to a globe with the shaded points being on the other side of the sphere. The figure is from the default viewpoint of Matlab. It is interesting to point out that obvious clusters of countries can be observed. For example, on the top left is the cluster of Commonwealth nations (Australia, Canada, India, and New Zealand). On the bottom right is the cluster of western allies (UK, US, and West Germany) with Japan not far on above of them. On the north pole is China, which reflects its isolated trading situation back in 1986. On the backside is the cluster of countries headed by USSR. On the left backside is the cluster of Brazil, Argentina, Egypt. We note that this figure appears different from those in $[10,13]$ mainly because that each used a different method on a different 


\section{Trading Globe of Countries}

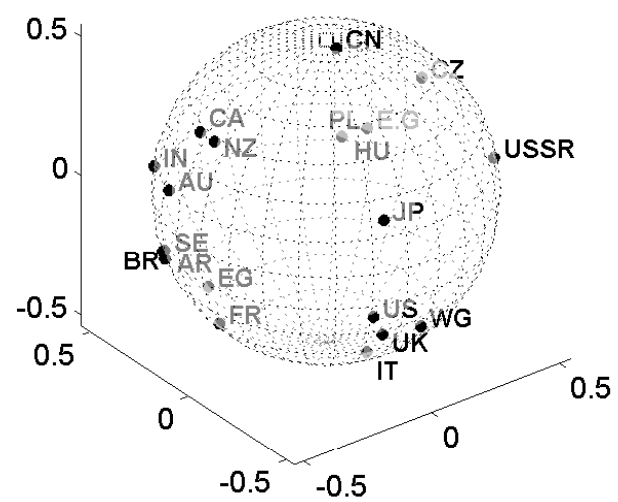

FIG. 5.2. Spherical representation for trading data in 1986 between countries $\{$ Argentina, Australia, Brazil, Canada, China, Czechoslovakia, East Germany, Egypt, France, Hungary, India, Italy, Japan, New Zealand, Poland, Sweden, UK, USA, USSR, West Germany\}.

(nonconvex) model of the spherical embedding of the data.

(E3) 3D Map of global cities in HA30 data set. HA30 is a dataset of spherical distances among 30 global cities, measured in hundreds of miles and selected by Hartigan [22] from the World Almanac, 1966. It also provides XYZ coordinates of those cities. In order to use FITS, we first convert the spherical distances to Euclidean distances through the formula: $d_{i j}:=2 R \sin \left(s_{i j} /(2 R)\right)$ where $s_{i j}$ is the spherical distance between city $i$ and city $j$ and $R=39.59$ (hundreds miles) is the Earth radius (see [38, Thm. 3.23]). The initial matrix $D^{0}$ consists of the squared distances $d_{i j}^{2}$. It is observed that the matrix $\left(-J D^{0} J\right)$ has 15 positive eigenvalues and 14 negative eigenvalues and 1 zero eigenvalue. Therefore, the original spherical distances are not accurate and contain large errors. Therefore, FITS is needed to correct those errors. We plot the resulting coordinates of the 30 cities in Fig. 5.3. One of the remarkable features is that FITS is able to recover the Earth radius with high accuracy $R=39.5916$.

We now assess the quality of the spherical embedding in Fig. 5.3 through a Procrustes analysis. Let $\bar{Y}$ be the final Euclidean distance matrix from FITS and let $X$ be obtained from (6). Let $x^{i}$ denotes the $i$ th column of $X$. Then, $x^{n+1}$ is the center of the sphere. We shift the center to origin and the resulting points are $z^{i}:=x^{i}-x^{n+1}$. Let $Z$ denote the matrix of consisting of $z^{i}$ as its columns. The data set HA30 includes a set of XZY coordinates of those 30 cities. We let $A$ denote those coordinates (in columns). We would like to see how close $Z$ and $A$ are. This can be done through solving the orthogonal Procrustes problem:

$$
\min _{P \in \mathbb{R}^{r \times r}} f=\|P Z-A\| \text {, s.t. } P^{T} P=I,
$$

which seeks the best rotational (including rotations and flips) matrix $P$ such that the columns in $Z$ best match the corresponding columns in $A$ after the rotation. Problem (56) has a closed form solution $P=U V^{T}$, where $U$ and $V$ are from the singularvalue-decomposition of $A Z^{T}=U S V^{T}$ with the standard meaning of $U, S$, and $V$. 


\section{D Map of 30 Global Cities}

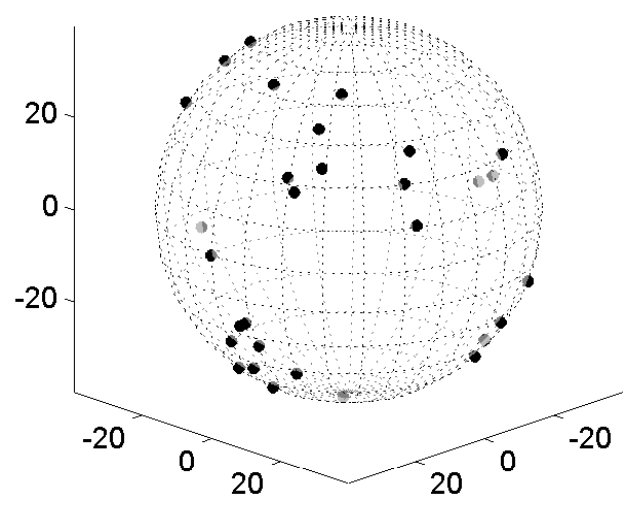

FIG. 5.3. Spherical embedding of HA30 data set with radius $R=39.5916$.

The optimal objective is $f=0.2782$. This small error is probably due to the fact that the radius used in HA30 is 39.59 in contrast to ours 39.5916. This small value also confirms the good quality of the embedding from FITS when compared to the solution in HA30.

(E4) Circle fitting. The problem of circle fitting has recently been studied in [2], where more references on the topic can be found. Let points $\left\{a^{i}\right\}_{i=1}^{n}$ with $a^{i} \in \mathbb{R}^{r}$ be given. The problem is to find a circle with center $x \in \mathbb{R}^{r}$ and radius $R$ such that the points stay as close to the circle as possible. Two criteria were considered in [2]:

$$
\min _{x, R} f_{1}=\sum_{i=1}^{n}\left(\left\|a^{i}-x\right\|-R\right)^{2}
$$

and

$$
\min _{x, R} f_{2}=\sum_{i=1}^{n}\left(\left\|a^{i}-x\right\|^{2}-R^{2}\right)^{2}
$$

Problem (58) is much easier to solve than (57). But the key numerical message in [2] is that (57) may produce far better geometric fitting than (58). This was demonstrated through the following example [2, Example 5.3]:

$$
a^{1}=\left[\begin{array}{l}
1 \\
9
\end{array}\right], a^{2}=\left[\begin{array}{l}
2 \\
7
\end{array}\right], a^{3}=\left[\begin{array}{l}
5 \\
8
\end{array}\right], a^{4}=\left[\begin{array}{l}
7 \\
7
\end{array}\right], a^{5}=\left[\begin{array}{l}
9 \\
5
\end{array}\right], a^{6}=\left[\begin{array}{l}
3 \\
7
\end{array}\right] .
$$

Model (58) produces a very small circle, not truly reflecting the geometric layout of the data.

The Euclidean distance embedding studied in this paper provides an alternative model. Let $D_{i j}^{0}=\left\|a^{i}-a^{j}\right\|^{2}$ for $i=1, \ldots, n$ and $n=6, r=2$ in this example. Let $\bar{Y}$ be the final distance matrix from FITS and the embedding points in $X$ be obtained from (6). The first 6 columns $\left\{x^{i}\right\}_{i=1}^{6}$ of $X$ correspond to the known points $\left\{a^{i}\right\}_{i=1}^{6}$. 


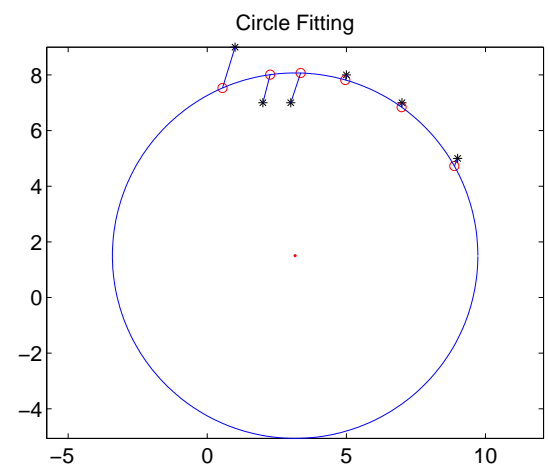

FIG. 5.4. Circle fitting of 6 points with $R=6.5673$. The known points and their corresponding points on the circle by FITS are linked by a line.

The last column $x^{7}$ is the center. The points $\left\{x^{i}\right\}_{i=1}^{6}$ are on the circle centered at $x^{7}$ with radius $R\left(R=\sqrt{\bar{Y}_{1(n+1)}}\right)$. We need to match $\left\{x^{i}\right\}_{i=1}^{6}$ to $\left\{a^{i}\right\}_{i=1}^{6}$ so that the known points stay as close to the circle as possible. This can be done through the orthogonal Procrustes problem (56).

We first centralize both sets of points. Let

$$
a^{0}:=\frac{1}{n} \sum_{i=1}^{n} a^{i}, \quad \bar{a}^{i}:=a^{i}-a^{0} \quad \text { and } \quad x^{0}:=\frac{1}{n} \sum_{i=1}^{n} x^{i}, \quad \bar{x}^{i}:=x^{i}-x^{0}, \quad i=1, \ldots, n .
$$

Let $A$ be the matrix whose columns are $\bar{a}^{i}$ and $Z$ whose columns are $\bar{x}^{i}$ for $i=1, \ldots, n$. Solve the orthogonal Procrustes problem (56) to get $P=U V^{T}$. The resulting points are

$$
z^{i}:=P \bar{x}^{i}+a^{0}, \quad i=1, \ldots, n
$$

and the new center, denoted by $z^{n+1}$, is

$$
z^{n+1}:=P\left(x^{n+1}-x^{0}\right)+a^{0} .
$$

It can be verified that the points $\left\{z^{i}\right\}_{i=1}^{n}$ are on the circle centered at $z^{n+1}$ with radius $R$. That is

$$
\left\|z^{i}-z^{n+1}\right\|^{2}=\left\|P\left(x^{i}-x^{n+1}\right)\right\|^{2}=\left\|x^{i}-x^{n+1}\right\|^{2}=R^{2} .
$$

This circle is the best circle from model (5) and is plotted in Fig. 5.4 with the pair of points $\left\{a^{i}, z^{i}\right\}$ being linked by a line. When the obtained center $x=z^{n+1}$ and $R$ are substituted to (57), we get $f_{1}=3.6789$, not far from the reported value $f_{1}=3.1724$ in [2]. The circle fits the original data reasonably well. We complete this example by noting a common feature between our model (5) and the squared least square model (58) in that the squared distances are used in both models. But the key difference is that (5) used all available pairwise squared distances among $a^{i}$ rather than just those from $a^{i}$ to the center $x$ as is in (58).

6. Conclusion. In this paper, we proposed a matrix optimization approach to the problem of Euclidean distance embedding on a sphere. We applied the majorized 
penalty method of Gao and Sun [17] to the resulting matrix problem. A key feature we exploited is that all subproblems to be solved share a common set of Euclidean distance constraints with a simple distance objective function. We showed that such problems can be efficiently solved by the Newton-CG method, which is proved to be quadratically convergent under constraint nondegeneracy.

Constraint nondegeneracy is a difficult constraint qualification to analyze. We proved it under a weak condition for our problem. We illustrated in Example 3.6 that this condition holds everywhere but one point $(t=0)$. This means that constraint nondegeneracy is satisfied for $t \neq 0$. For the case $t=0$, we can verify (through verifying Lemma 3.7) that constraint nondegeneracy also holds. This motivates our open question whether constraint nondegeneracy should hold under a weaker condition.

In the numerical part, we used 4 existing embedding problems on a sphere to demonstrate a variety of applications that the developed algorithm can be applied to. The first two examples are from classical MDS and new features (wheel representation for E1 and new clusters for E2) are revealed. For E3, despite the large noises in the initial distance matrix, our method is remarkably able to recover the Earth radius and to project accurate mapping of the 30 global cities on the sphere. The last example is different from the others in that its inputs are the coordinates of known points (rather than a distance matrix). Finding the best circle to fit those points requires localization of its center and radius. A Procrustes procedure is described to help finish the job. The resulting visualizations are very satisfactory for all the examples. Since those examples are of small scale, our method took less than 1 second to find the optimal embedding. Hence, we omitted reporting such information. In future, we plan to investigate its application in machine learning on manifolds, which would involve large data sets as well as higher dimensional embedding $(r>3)$.

Acknowledgements. We would like to thank the two referees for their valuable comments, especially on computing a particular generalized Jacobian matrix in the practical implementation of the Newton-CG method. The comments from the associate editor are also greatly appreciated.

\section{REFERENCES}

[1] F. Alizadeh, J.-P. A Haeberly, M.L. Overton, Complementarity and nondegeneracy in semidefinite programming, Math. Program. 77, 111-128 (1997)

[2] A. BECK AND D. PAN, On the solution of the GPS localization and circle fitting problems, SIAM J. Optm. 22 (2012), pp. 108-134.

[3] J.F. Bonnans and A. Shapiro, Perturbation Analysis of Optimization Problems, SpringerVerlag, New York, 2000.

[4] I. Borg And P.J.F. Groenen, Modern Multidimensional Scaling: Theory and Applications (2nd ed.) Springer Series in Statistics, Springer, 2005.

[5] I. Borg And J.C. Lingoes, A model and algorithm for multidimensional scaling with external constraints on the distances, Psychometrika 45 (1980), pp. 25-38.

[6] X. Chen, H.-D. QI, And P. Tseng, Analysis of nonsmooth symmetric matrix valued functions with applications to semidefinite complementarity problems, SIAM J. Optim. 13 (2003), pp. 960-985.

[7] Z.X. Chan AND D.F. Sun, Constraint nondegeneracy, strong regularity and nonsigularity in semidefinite programming, SIAM J. Optim. 19 (2008), 370-396.

[8] F.H. Clarke, Optimization and Nonsmooth Analysis, John Wiley \& Sons, New York, 1983.

[9] T.F. Cox And M.A.A. Cox, Multidimensional Scaling, 2nd Ed, Chapman and Hall/CRC, 2001.

[10] T.F. Cox And M.A.A. Cox, Multidimensional scaling on a sphere, Commu. Statist. - Theory Meth., 20 (1991), pp. 2943-2953. 
[11] G. Crippen and T. Havel, Distance Geometry and Molecular Conformation, New York: Wiley, 1988.

[12] J. Dattorro, Convex Optimization and Euclidean Distance Geometry, Meboo Publishing USA, 2005.

[13] J. DE LEeUw AND P. MAIR, Multidimensional scaling using majorization: SMACOF in R, J. Stat. Software 31 (2009), pp. 1-30.

[14] G. Ekman, Dimensions of color vision, Journal of Psychology 38 (1954), pp. 467-474.

[15] N. Gaffke and R. Mathar, A cyclic projection algorithm via duality, Metrika, 36 (1989), pp. 29-54.

[16] Y. GAO, Structured Low Rank Matrix Optimization Problems: a Penalty Approach, PhD Thesis (2010), National University of Singapore.

[17] Y. GAO AND D.F. SUN, A majorized penalty approach for calibrating rank constrained correlation matrix problems. Technical Report, Department of Mathematics, National University of Singapore, March 2010.

[18] W. Glunt, T.L. Hayden, S. Hong, and J. Wells, An alternating projection algorithm for computing the nearest Euclidean distance matrix, SIAM J. Matrix Anal. Appl., 11 (1990), pp. 589-600.

[19] W. Glunt, T.L. Hayden, and R. Raydan, Molecular conformations from distance matrices, J. Computational Chemistry, 14 (1993), pp. 114-120.

[20] J.C. GoweR, Some distance properties of latent root and vector methods in multivariate analysis, Biometrika, 53 (1966), pp. 315-328.

[21] J.C. Gower, Properties of Euclidean and non-Euclidean distance matrices, Linear Algebra Appl., 67 (1985), pp. 81-97.

[22] J. Hartigan, Clustering Algorithms, Wiley, 1975,

[23] J.-B. Hiriart-Urruty and C. Lemaréchal, Convex Analysis and Minimization Algorithms I, Springer-Verlag, Berlin 1993.

[24] J.-B. Hiriart-Urruty, J.-J. Strodiot, and V.H. Nguyen, Generalized Hessian matrix and second-order optimality conditions for problems with $C^{1,1}$ data, Appl. Math. Optim. 11 (1984), pp. 43-56.

[25] B. Jiang And Y.-H. DAI, A framework of constraint preserving update schemes for optimization on the Stiefel manifold, Tech. Report, Academy of Mathematical Sciences, Chinese Academy of Sciences, Dec. 2012.

[26] M Laurent and A. Varvitsiotis, Positive semidefinite matrix completion using rigid and the strong Arnold property, Linear Alg. Appl. 452 (2014), pp. 292-317.

[27] C. Lavor, L. Liberti, N. Maculan, and A. Mucherino, Recent advances on the discretization molecular distance geometry problem, European J. Oper. Res. 219 (2012), pp. 698706.

[28] S.-Y. Lee And P.M. Bentler, Functional relations in multidimensional scaling, British J. Math. Stat. Psychology 33 (1980), pp. 142-150.

[29] Q.-N. Li AND H.-D. QI, A sequential semismooth Newton method for the nearest low-rank correlation matrix problem, SIAM J. Optim. 21 (2011), pp. 1641-1666.

[30] L. Liberti, C. Lavor, N. Maculan, and A. Mucherino, Euclidean distance geometry and applications, SIAM Rev. 56-1 (2014), pp. 3-69.

[31] C. Ling, J. Nie, L. QI AND Y. YE, Biquadratic optimization over unit spheres and semidefinite programming relaxations, SIAM J. Optim. 20 (2009), pp. 1286-1310.

[32] J. MALICK, The spherical constraint in Boolean quadratic programs, J. Glob. Optim. 39 (2007), pp. 609-622.

[33] W. MiaO, S. Pan, And D.F. Sun, A rank-corrected procedure for matrix completion with fixed basis coefficients, Arxiv preprint arXiv:1210.3709, 2012.

[34] W. MiaO, Matrix completion procedure with fixed basis coefficients and rank regularized problems with hard constraints, PhD thesis (2013), Department of Mathematics, National University of Singapore.

[35] B. Mishra, G. Meyer, and R. Sepulchre, Low-rank optimization for distance matrix completion. In Proc. of 50th IEEE Con. Decis. Cont., December 2011.

[36] J.J. Moré AND Z. Wu, Distance geometry optimization for protein structures, J. Global Optimization 15 (1999), pp. 219-234.

[37] J.-S. Pang, D.F. Sun, And J. Sun, Semismooth homeomorphisms and strong stability of semidefinite and Lorentz complementarity problems, Math. Oper. Res. 28 (2003), pp. 39-63.

[38] E. Pȩkalaska and R.P.W. Duin The Dissimilarity Representation for Pattern Recognition: Foundations and Application, Series in Machine Perception Artificial Intelligence 64, World Scientific 2005. 
[39] H.-D. QI, A semismooth Newton method for the nearest Euclidean distance matrix problem, SIAM J. Matrix Anal. Appl. 34 (2013), pp. 67-93.

[40] H.-D. QI AND D.F. Sun, A quadratically convergent Newton method for computing the nearest correlation matrix, SIAM J. Matrix Anal. Appl. 28 (2006), pp. 360-385.

[41] H.-D. QI AND X.M. YuAN, Computing the nearest Euclidean distance matrix with low embedding dimensions, Math. Program. 147 (2014), pp. 351-389.

[42] L. Qi And J. Sun, A nonsmooth version of Newton's method, Math. Program. 58 (1993), pp. 353-367.

[43] S.M. Robinson, Local structural of feasible sets in nonlinear programming, part III: Stability and sensitivity, Math. Programming Stud. 30 (1987), pp. 45-66.

[44] S.M. Robinson, Constraint nondegeneracy in variational analysis, Math. Oper. Res. 28 (3003), 201-232.

[45] P. Schönemann, A generalized solution of the orthogonal procrustes problem, Psychometrika, 31 (1966), pp. 1-10.

[46] I.J. Schomngerg, Remarks to Maurice Fréchet's article "Sur la définition axiomatque d'une classe d'espaces vectoriels distanciés applicbles vectoriellement sur l'espace de Hilbet", Ann. Math. 36 (1935), pp. 724-732.

[47] I.J. SchoenBerG, On certain metric spaces arising from Euclidean spaces by a change of metric and their embedding in Hilbert space, Ann. Math. 38 (1938), pp. 787-797.

[48] A. ShapIro, Sensitivity analysis of generalized equations, J. Math. Sci. 115 (2003), pp. 25542565.

[49] D.F. Sun, The strong second-order sufficient condition and constraint nondegeneracy in nonlinear semidefinite programming and their implications, Math. Oper. Res. 31, 761-776 (2006)

[50] D.F. Sun and J. Sun, Semismooth matrix valued functions, Math. Oper. Res. 27 (2002), pp. 150-169.

[51] K.C. ТоH, An inexact path-following algorithm for convex quadratic SDP, Math. Program. 112 (2008), pp. 221-254.

[52] Z.-W. Wen AND W.-T. Yin, A feasible method for optimization with orthogonal constraints, Math. Programming, 142 (2013), pp. 397-434..

[53] G. Young And A.S. Householder, Discussion of a set of points in terms of their mutual distances, Psychometrika 3 (1938), pp. 19-22.

[54] X.Y. Zhao, D.F. Sun, And K.-C. ToH, A Newton-CG augmented Lagrangian method for semidefinite programming, SIAM J. Optim. 20 (2010), pp. 1737-1765.

[55] G. Zhou, L. Caccetta, K.-L. Teo and S. Wu, Nonnegative polynomial optimization over unit spheres and convex programming relaxations, SIAM J. Optim. 22 (2012), pp. 9871008. 